\title{
Coordinate-space solution of the Skyrme-Hartree-Fock-Bogolyubov equations within spherical symmetry. The program HFBRAD (v1.00)
}

\author{
K. Bennaceur* \\ IPN Lyon, CNRS-IN2P3/UCB Lyon 1, Bât. Paul Dirac, \\ 43, Bd. du 11 novembre 1918, 69622 Villeurbanne Cedex, France \\ J. Dobaczewski \\ Institute of Theoretical Physics, Warsaw University \\ ul. Hoża 69, PL-00681 Warsaw, Poland \\ Department of Physics and Astronomy, The University of Tennessee, \\ Knoxville, Tennessee 37996, USA \\ Physics Division, Oak Ridge National Laboratory, \\ P.O. Box 2008, Oak Ridge, Tennessee 37831, USA
}

\begin{abstract}
We describe the first version (v1.00) of the code HFBRAD which solves the SkyrmeHartree-Fock or Skyrme-Hartree-Fock-Bogolyubov equations in the coordinate representation within the spherical symmetry. A realistic representation of the quasiparticle wave functions on the space lattice allows for performing calculations up to the particle drip lines. Zero-range density-dependent interactions are used in the pairing channel. The pairing energy is calculated by either using a cut-off energy in the quasiparticle spectrum or the regularization scheme proposed by A. Bulgac and Y. Yu.
\end{abstract}

Key words: Hartree-Fock, Hartree-Fock-Bogolyubov, Skyrme interaction, Self-consistent mean-field, Nuclear many-body problem; Pairing, Nuclear radii, Single-particle spectra, Coulomb field

PACS: 07.05.T, 21.60.-n, 21.60.Jz

\footnotetext{
* Corresponding author.

Email addresses: bennaceur@ipnl.in2p3.fr (K. Bennaceur), jacek.dobaczewski@fuw.edu.pl (J. Dobaczewski).
} 


\section{PROGRAM SUMMARY}

Title of the program: HFBRAD (v1.00)

Program obtainable from: CPC Program Library, Queen's University of Belfast, N. Ireland

Licensing provisions: none

Computers on which the program has been tested: Pentium-III, Pentium-IV

Operating systems: LINUX, Windows

Programming language used: FORTRAN-95

Memory required to execute with typical data: 30 MBytes

No. of bits in a word: The code is written with a type real corresponding to 32-bit on any machine. This is achieved using the intrinsic function selected_real_kind at the beginning of the code and asking for at least 12 significant digits. This can be easily modified by asking for more significant digits if the architecture of the computer can handle it.

No. of processors used: 1

Has the code been vectorised?: No.

No. of bytes in distributed program, including test data, etc.: 400 kbytes

No. of lines in distributed program: 5164 (of which 1635 are comments and separators)

Nature of physical problem: For a self-consistent description of nuclear pair correlations, both the particle-hole (field) and particle-particle (pairing) channels of the nuclear mean field must be treated within the common approach, which is the Hartree-Fock-Bogolyubov theory. By expressing these fields in spatial coordinates one can obtain the best possible solutions of the problem; however, without assuming specific symmetries the numerical task is often too difficult. This is not the case when the spherical symmetry is assumed, because then the one-dimensional differential equations can be solved very efficiently. Although the spherically symmetric solutions are physically meaningful only for magic and semi-magic nuclei, the possibility of obtaining them within tens of seconds of the CPU makes them a valuable element of studying nuclei across the nuclear chart, including those near or at the drip lines.

Method of solution: The program determines the two-component HartreeFock-Bogolyubov quasiparticle wave functions on the lattice of equidistant 
points in the radial coordinate. This is done by solving the eigensystem of two second-order differential equations by using the Numerov method. Standard iterative procedure is then used to find self-consistent solutions for the nuclear product wave functions and densities.

Restrictions on the complexity of the problem: The main restriction is related to the assumed spherical symmetry.

Typical running time: One Hartree-Fock iteration takes about $0.4 \mathrm{sec}$ for a medium mass nucleus, convergence is achieved in about $40 \mathrm{sec}$.

Unusual features of the program: none

\section{LONG WRITE-UP}

\section{Introduction}

For several decades, the ground state of nuclei have been studied within the self-consistent mean-field approximation. Such a description of the atomic nucleus, has ability to properly account for the bulk properties of nuclei such as masses, radii or shape. The Hartree-Fock method provides a good approximation of closed shell magic nuclei, however, the pairing correlations constitute an essential ingredient for the description of open shell nuclei. These effects are usually described by the Hartree-Fock plus BCS (HFBCS) or Hartree-FockBogolyubov (HFB) methods.

Two main classes of numerical methods have been so far used for the implementation of the HFBCS or HFB methods. In the first one, one employs the expansion of the quasiparticle states on a discrete basis of orthogonal functions, usually provided by the harmonic oscillator potential. In this case the non-linear $\mathrm{HFB}(\mathrm{CS})$ equations are formulated in a matrix form and can be solved by using an iterative procedure (hereafter Bogolyubov iterations). In the second class, one uses the direct integration of the equations in the coordinate representation. This is usually done within the box boundary conditions to discretize the spectrum of quasiparticle states. The first approach is more elegant and efficient for finite-range interactions, however, it has a disadvantage that the use of harmonic oscillator wave functions may perturb the correct description of the asymptotics of the system. On the other hand, the asymptotic part of the quasiparticle wave functions does not suffer from this 
possible weakness when the problem is solved in the coordinate basis, but here one has to solve a set of integro-differential equations and as a consequence this method is usually only applied for the zero-range Skyrme force, for which the problem reduces to a set of differential equations.

The question of the asymptotic properties of the atomic nuclei (neutron skins, halos) is an urgent challenge in theoretical nuclear structure, and is particularly important due to the availability of beams of exotic nuclei ${ }^{1}$, which allow for relevant experiments. The HFB problem in coordinate basis, along with the use of the effective Skyrme force is indeed a powerful tool for studying ground-state properties of nuclei, and such studies have been and will be pursued especially in the regions of nuclear chart where the experiments are being performed.

Two computer codes that solve self-consistent equations on the basis have recently been published $[1,2]$ for non-spherical shapes. We refer the reader to these publications for a review of approaches and methods that can be and have been used in such cases. Within the assumed spherical symmetry, which is the subject of the present study, the first self-consistent solutions were obtained in the 1970's [3,4], and these old codes were later used for decades by different groups, however, they have never been published.

The first description and implementation of the HFB problem within the coordinate-space spherical symmetry was presented in Ref. [5]. The code written during this work was later updated many times since its early version, and distributed widely, however, neither this code was ever the object of an official publication. Indeed, the absence of accompanying manual makes it hard to use, and its older versions are sometimes used despite the fact that the newer ones have been made available. For coordinate-space spherical symmetry, the code solving the HFBCS equations for the Skyrme interaction was published in Ref. [6] and that for the relativistic mean field Hartree-Bogolyubov method in Ref. [7]. A similar unpublished code also exists to treat pairing correlations for the finite-range Gogny interaction [8], although the code that would solve the full HFB problem within these conditions is not yet available.

The aim of this work is to provide a modern code (named HFBRAD) for the spherical Skyrme-HFB problem in the coordinate representation. The code constitutes a completely rewritten version of that constructed in Ref. [5], but it also features implementation of the pairing renormalization [9]. Although such code has to be used with caution in open-shell nuclei, because there the deformation effects not included here are essential, it nevertheless provides a fast and easy tool for a quick estimate of nuclear properties, which can precede much more time consuming calculations beyond the spherical symme-

1 For example in Europe: ARENAS3, Louvain-la-Neuve; ISOLDE-CERN, Geneva; GANIL, Caen; and GSI, Darmstadt. 
try. In this paper we also provide a clear description of the different Skyrme forces implemented in the code, terms neglected in the energy functional, and parameters in the pairing channel.

This paper is organized as follows. In section 2 we briefly show how the SkyrmeHFB equations are derived and discuss their main properties. Notably, a significant part is devoted to the problem of the divergence of the energy due to the zero range of the force in the pairing channel. In section 3 we present several parametrizations of the Skyrme force implemented in the code, with a particular attention paid to the pairing channel. In section 4 we give definitions and meanings of the various quantities and observables which are the result of calculations. Some aspects of the numerical treatment of the problem are presented in section 5, and finally, sections 6 and 7 give a precise description of the input and output data files.

\section{The Skyrme Hartree-Fock-Bogolyubov equations}

The HFB approximation is based on the use of a trial variational wave function which is assumed to be an independent quasiparticle state $|\Phi\rangle$. This state, which mixes different eigenstates of the particle number operator, is a linear combination of independent particle states representing various possibilities of occupying pairs of single particle states. Following the notations and phase convention of [5] we define the particle and pairing density $\rho$ and $\tilde{\rho}$ matrices by

$$
\begin{gathered}
\rho\left(\mathbf{r} \sigma q, \mathbf{r}^{\prime} \sigma^{\prime} q^{\prime}\right)=\left\langle\Phi\left|a_{\mathbf{r}^{\prime} \sigma^{\prime} q^{\prime}}^{\dagger} a_{\mathbf{r} \sigma q}\right| \Phi\right\rangle, \\
\tilde{\rho}\left(\mathbf{r} \sigma q, \mathbf{r}^{\prime} \sigma^{\prime} q^{\prime}\right)=-2 \sigma^{\prime}\left\langle\Phi\left|a_{\mathbf{r}^{\prime}-\sigma^{\prime} q^{\prime}} a_{\mathbf{r} \sigma q}\right| \Phi\right\rangle,
\end{gathered}
$$

where the operators $a_{\mathbf{r} \sigma q}^{\dagger}$ and $a_{\mathbf{r} \sigma q}$ create and annihilate a nucleon at the point $\mathbf{r}$ having spin $\sigma= \pm \frac{1}{2}$ and isospin $q= \pm \frac{1}{2}$. The symmetry properties of $\rho$ and $\tilde{\rho}$ as well as the relation between $\tilde{\rho}$ and the pairing tensor $\kappa$ (defined for example in [10]) are discussed in [5].

The variation of the energy expectation value $E=\langle\Phi|\hat{H}| \Phi\rangle$ with respect to $\rho$ and $\tilde{\rho}$ under the constraints $N=\langle\Phi|\hat{N}| \Phi\rangle$ and $Z=\langle\Phi|\hat{Z}| \Phi\rangle$ (for neutrons and protons) leads to the Hartree-Fock-Bogolyubov equation which reads in 
coordinate representation

$$
\begin{aligned}
& \int d^{3} \mathbf{r}^{\prime} \sum_{\sigma^{\prime}}\left(\begin{array}{cc}
h\left(\mathbf{r} \sigma, \mathbf{r}^{\prime} \sigma^{\prime}\right) & \tilde{h}\left(\mathbf{r} \sigma, \mathbf{r}^{\prime} \sigma^{\prime}\right) \\
\tilde{h}\left(\mathbf{r} \sigma, \mathbf{r}^{\prime} \sigma^{\prime}\right)-h\left(\mathbf{r} \sigma, \mathbf{r}^{\prime} \sigma^{\prime}\right)
\end{array}\right)\left(\begin{array}{c}
\varphi_{1}\left(E, \mathbf{r}^{\prime} \sigma^{\prime}\right) \\
\varphi_{2}\left(E, \mathbf{r}^{\prime} \sigma^{\prime}\right)
\end{array}\right)= \\
&\left(\begin{array}{cc}
E+\lambda & 0 \\
0 & E-\lambda
\end{array}\right)\left(\begin{array}{l}
\varphi_{1}(E, \mathbf{r} \sigma) \\
\varphi_{2}(E, \mathbf{r} \sigma)
\end{array}\right)
\end{aligned}
$$

where the particle and pairing fields are given by

$$
h\left(\mathbf{r} \sigma, \mathbf{r}^{\prime} \sigma^{\prime}\right)=\frac{\delta E}{\delta \rho\left(\mathbf{r} \sigma, \mathbf{r}^{\prime} \sigma^{\prime}\right)}, \quad \tilde{h}\left(\mathbf{r} \sigma, \mathbf{r}^{\prime} \sigma^{\prime}\right)=\frac{\delta E}{\delta \tilde{\rho}\left(\mathbf{r} \sigma, \mathbf{r}^{\prime} \sigma^{\prime}\right)} .
$$

Once again we refer the reader to the article [5] for the discussion concerning the quasiparticle spectrum, its symmetries and the relations between the components of the HFB spinors and the densities.

\subsection{Local densities}

In the Skyrme-HFB formalism, the evaluation of the expectation value of the energy leads to an expression which is a functional of the local densities, namely, the particle (normal) and pairing (abnormal) densities

$$
\rho(r)=\sum_{i} \varphi_{2}\left(E_{i}, r\right)^{2} \quad \text { and } \quad \tilde{\rho}(r)=-\sum_{i} \varphi_{1}\left(E_{i}, r\right) \varphi_{2}\left(E_{i}, r\right),
$$

and their derivatives. The presence of non local terms in the force leads to a dependence on the normal and abnormal kinetic densities

$$
\begin{aligned}
& \tau(\mathbf{r})=\sum_{i}\left|\boldsymbol{\nabla} \varphi_{2}\left(E_{i}, \mathbf{r}\right)\right|^{2} \\
& \tilde{\tau}(\mathbf{r})=-\sum_{i} \boldsymbol{\nabla} \varphi_{1}\left(E_{i}, \mathbf{r}\right) \cdot \boldsymbol{\nabla} \varphi_{2}\left(E_{i}, \mathbf{r}\right),
\end{aligned}
$$

while the spin-orbit term leads to a dependence on the spin current tensors $\mathbb{J}_{i j}$ and $\tilde{\mathbb{J}}_{i j}$. We do not give here the definitions of these tensors, because for the spherical symmetry discussed here they reduce to the corresponding spin current vectors $\mathbf{J}$ and $\tilde{\mathbf{J}}$

$$
\begin{aligned}
& \mathbf{J}(\mathbf{r})=\mathrm{i} \sum_{i} \varphi_{2}\left(E_{i}, \mathbf{r}\right) \boldsymbol{\nabla} \varphi_{2}\left(E_{i}, \mathbf{r}\right)\left\langle\sigma^{\prime}|\hat{\boldsymbol{\sigma}}| \sigma\right\rangle, \\
& \tilde{\mathbf{J}}(\mathbf{r})=-\mathrm{i} \sum_{i} \varphi_{1}\left(E_{i}, \mathbf{r}\right) \boldsymbol{\nabla} \varphi_{2}\left(E_{i}, \mathbf{r}\right)\left\langle\sigma^{\prime}|\hat{\boldsymbol{\sigma}}| \sigma\right\rangle .
\end{aligned}
$$




\subsection{Spherical symmetry}

In the code HFBRAD the solutions are restricted to have a spherical symmetry and do not mix proton and neutron states. In this situation the wave functions have the good quantum numbers $(n \ell j m q)$ ( $n$ is a good quantum number since the spectrum is discretized inside a spherical box) and all the solutions inside an $(n \ell j q)$-block are degenerated. Furthermore, the radial part of the wave functions can be chosen to be real. Thus we use the ansatz

$$
\varphi_{i}(E, \mathbf{r} \sigma)=\frac{u_{i}(n \ell j, r)}{r} Y_{m_{\ell}}^{(\ell)}(\hat{r})\left\langle\ell m_{\ell} \frac{1}{2} \sigma \mid j m\right\rangle, \quad i=1,2
$$

for the wave functions. The local densities can be written using the radial functions (omitting the isospin quantum number)

$$
\begin{aligned}
& \rho(r)=\frac{1}{4 \pi r^{2}} \sum_{n \ell j}(2 j+1) u_{2}^{2}(n \ell j, r), \\
& \tilde{\rho}(r)=-\frac{1}{4 \pi r^{2}} \sum_{n \ell j}(2 j+1) u_{1}(n \ell j, r) u_{2}(n \ell j, r) .
\end{aligned}
$$

For the kinetic densities we have

$$
\begin{aligned}
\tau(r)= & \sum_{n \ell j} \frac{2 j+1}{4 \pi r^{2}}\left[\left(u_{2}^{\prime}(n \ell j, r)-\frac{u_{2}(n \ell j, r)}{r}\right)^{2}+\frac{\ell(\ell+1)}{r^{2}} u_{2}^{2}(n \ell j, r)\right],(13) \\
\tilde{\tau}(r)= & -\sum_{n \ell j} \frac{2 j+1}{4 \pi r^{2}}\left[\left(u_{1}^{\prime}(n \ell j, r)-\frac{u_{1}(n \ell j, r)}{r}\right)\left(u_{2}^{\prime}(n \ell j, r)-\frac{u_{2}(n \ell j, r)}{r}\right)\right. \\
& \left.+\frac{\ell(\ell+1)}{r^{2}} u_{1}(n \ell j, r) u_{2}(n \ell j, r)\right]
\end{aligned}
$$

Finally the spin current vector densities have only one non vanishing component given by

$$
\begin{aligned}
& J(r)=\frac{1}{4 \pi r^{3}} \sum_{n \ell j}(2 j+1)\left[j(j+1)-\ell(\ell+1)-\frac{3}{4}\right] u_{2}^{2}(n \ell j, r), \\
& \tilde{J}(r)=-\frac{1}{4 \pi r^{3}} \sum_{n \ell j}(2 j+1)\left[j(j+1)-\ell(\ell+1)-\frac{3}{4}\right] u_{1}(n \ell j, r) u_{2}(n \ell j, r \ell
\end{aligned}
$$




\subsection{The Hartree-Fock-Bogolyubov energy}

In the Skyrme-HFB approximation, the total energy $\mathcal{E}$ of a nucleus is given as a sum of kinetic, Skyrme, pairing and Coulomb terms:

$$
\begin{aligned}
E & =K+E_{\text {Skyrme }}+E_{\text {pair }}+E_{\text {Coul }} \\
& =\int d^{3} \mathbf{r}\left[\mathcal{K}(\mathbf{r})+\mathcal{E}_{\text {Skyrme }}(\mathbf{r})+\mathcal{E}_{\text {pair }}(\mathbf{r})+\mathcal{E}_{\text {Coul }}(\mathbf{r})\right] .
\end{aligned}
$$

The derivation of the energy is explained in detail in several articles (see Refs. $[3,11]$ for the HF energy and [5] for the HFB case). The expression of the Skyrme force is given in section 3 .

In equation (17), the kinetic energy of both neutrons and protons is given by isoscalar kinetic density. The neutron and proton masses being approximated by their average value, and one has

$$
\mathcal{K}=\frac{\hbar^{2}}{2 m} \tau\left(1-\frac{1}{A}\right)
$$

where the factor in parentheses takes into account the direct part of the centerof-mass correction [12]. Since we only consider even-even nuclei, the Skyrme part of the energy functional is time even, it can be written as a sum of isoscalar $(T=0)$ and isovector $(T=1)$ parts (see e.g. Ref. [13]) or as a sum on isoscalar, neutrons and protons densities,

$$
\begin{aligned}
\mathcal{E}_{\text {Skyrme }} & =\frac{1}{2} t_{0}\left[\left(1+\frac{x_{0}}{2}\right) \rho^{2}-\left(x_{0}+\frac{1}{2}\right) \sum_{q} \rho_{q}^{2}\right] \\
& +\frac{t_{1}}{4}\left\{\left(1+\frac{x_{1}}{2}\right)\left[\rho \tau+\frac{3}{4}(\nabla \rho)^{2}\right]-\left(x_{1}+\frac{1}{2}\right) \sum_{q}\left[\rho_{q} \tau_{q}+\frac{3}{4}\left(\nabla \rho_{q}\right)^{2}\right]\right\} \\
& +\frac{t_{2}}{4}\left\{\left(1+\frac{x_{2}}{2}\right)\left[\rho \tau-\frac{1}{4}(\nabla \rho)^{2}\right]+\left(x_{2}+\frac{1}{2}\right) \sum_{q}\left[\rho_{q} \tau_{q}-\frac{1}{4}\left(\nabla \rho_{q}\right)^{2}\right]\right\} \\
& -\frac{1}{16}\left(t_{1} x_{1}+t_{2} x_{2}\right) J^{2}+\frac{1}{16}\left(t_{1}-t_{2}\right) \sum_{q} J_{q}^{2} \\
& +\frac{1}{12} t_{3} \rho^{\gamma}\left[\left(1+\frac{x_{3}}{2}\right) \rho^{2}-\left(x_{3}+\frac{1}{2}\right) \sum_{q} \rho_{q}^{2}\right] \\
& +\frac{1}{2} W_{0}\left(J \nabla \rho+\sum_{q} J_{q} \nabla \rho_{q}\right)
\end{aligned}
$$

Index $q$ stands for neutrons and protons while the absence of index indicates the total (isoscalar) density. Using the same notations for the densities, the pairing energy density reads 


$$
\begin{aligned}
\mathcal{E}_{\text {pair }}=\sum_{q}\left\{\frac{t_{0}^{\prime}}{4}\left(1-x_{0}^{\prime}\right) \tilde{\rho}_{q}^{2}+\frac{t_{1}^{\prime}}{4}\left(1-x_{1}^{\prime}\right)\left[\tilde{\rho}_{q} \tilde{\tau}_{q}+\frac{1}{4}\left(\nabla \tilde{\rho}_{q}\right)^{2}\right]\right. \\
\left.+\left[\frac{t_{2}^{\prime}}{8}\left(1+x_{2}^{\prime}\right)+\frac{1}{4} W_{0}^{\prime}\right] \tilde{J}_{q}^{2}+\frac{t_{3}^{\prime}}{24}\left(1-x_{3}^{\prime}\right) \rho^{\gamma^{\prime}} \tilde{\rho}_{q}^{2}\right\}
\end{aligned}
$$

In this last expression we have added a prime to the parameters of the interaction. Although the derivation of the general Skyrme energy density functional is based on a unique force, its effective nature justifies the use of different sets of parameters in the particle-hole and particle-particle channels. The prime indices anticipates this possibility which is discussed in section 3 .

The energy density defined in (17) involves a Coulomb term for protons. This term contains a direct part which can be expressed using the charge density and leads to a local field after variation of the energy and an exchange part which would lead to a non local potential. Both parts deserve a special discussion. The direct part of the Coulomb energy depends on the charge density $\rho_{c h}(\mathbf{r})$ and reads

$$
\mathcal{E}_{\text {coul }}^{\operatorname{dir}}=\frac{e^{2}}{2} \iint d^{3} \mathbf{r} d^{3} \mathbf{r}^{\prime} \frac{\rho_{c h}(\mathbf{r}) \rho_{c h}\left(\mathbf{r}^{\prime}\right)}{\left|\mathbf{r}-\mathbf{r}^{\prime}\right|}
$$

We use the point proton density to simplify this expression, i.e. the charge density is replaced by the proton density $\rho_{p}(\mathbf{r})$. Nevertheless, the proton form factor is taken into account when we calculate the charge radius of the nucleus (see eq. (57)). The exchange part of the Coulomb energy leads to a non local term and is treated with the Slater approximation. This approximation means that we keep only the first term of the density matrix expansion in the local density approximation [14]

$$
\mathcal{E}_{\mathrm{coul}}^{\mathrm{ex}}=-\frac{3}{4} e^{2}\left(\frac{3}{\pi}\right)^{\frac{1}{3}} \int d^{3} \mathbf{r} \rho_{p}^{4 / 3}(\mathbf{r}) .
$$

The error introduced by this approximation has been estimated by studying the next order term [15] or more recently by comparison with the exact treatment of the Coulomb energy [16]. The Slater approximation seems to have little consequence on the nuclei bulk properties although some significant effects can be expected on the position of the proton drip-line and the Coulomb displacement energy of single particle levels. Finally, the Coulomb contributions to the pairing energy and fields are not included in the HFBRAD code. 


\subsection{The Hartree-Fock-Bogolyubov mean fields}

Since the Bogolyubov transformation does not preserve the particle number, we introduce two Lagrange multipliers $\lambda_{N}$ and $\lambda_{Z}$ to conserve the average neutron and proton number. The HFB equations are then obtained by writing the stationary condition $\delta\left[\mathcal{E}-\left\langle\lambda_{N} N+\lambda_{Z} Z\right\rangle\right]=0$. The dependence of $\mathcal{E}$ on the kinetic densities leads the effective mass

$$
\begin{aligned}
& M_{q}= \frac{\hbar^{2}}{2 m_{q}^{*}} \\
&=\frac{\hbar^{2}}{2 m}+\frac{t_{1}}{4}\left[\left(1+\frac{x_{1}}{2}\right) \rho-\left(x_{1}+\frac{1}{2}\right) \rho_{q}\right] \\
& \quad+\frac{t_{2}}{4}\left[\left(1+\frac{x_{2}}{2}\right) \rho+\left(x_{1}+\frac{1}{2}\right) \rho_{q}\right]
\end{aligned}
$$

and to the abnormal effective mass

$$
\tilde{M}_{q}=\frac{t_{1}^{\prime}}{4}\left(1-x_{1}^{\prime}\right) \tilde{\rho}_{q} .
$$

The particle-hole (Hartree-Fock) fields is given by

$$
\begin{aligned}
U_{q} & =t_{0}\left[\left(1+\frac{x_{0}}{2}\right) \rho-\left(x_{0}+\frac{1}{2}\right) \rho_{q}\right] \\
& +\frac{t_{1}}{4}\left[\left(1+\frac{x_{1}}{2}\right)\left(\tau-\frac{3}{2} \Delta \rho\right)-\left(x_{1}+\frac{1}{2}\right)\left(\tau_{q}-\frac{3}{2} \Delta \rho_{q}\right)\right] \\
& +\frac{t_{2}}{4}\left[\left(1+\frac{x_{2}}{2}\right)\left(\tau+\frac{1}{2} \Delta \rho\right)+\left(x_{2}+\frac{1}{2}\right)\left(\tau_{q}+\frac{1}{2} \Delta \rho_{q}\right)\right] \\
& +\frac{t_{3}}{12}\left[\left(1+\frac{x_{3}}{2}\right)(2+\gamma) \rho^{\gamma+1}-\left(x_{3}+\frac{1}{2}\right)\left(\gamma \rho^{\gamma-1} \sum_{q^{\prime}} \rho_{q^{\prime}}^{2}+2 \rho^{\gamma} \rho_{q}\right)\right] \\
& +\frac{t_{3}^{\prime}}{24}\left(1-x_{3}^{\prime}\right) \gamma^{\prime} \rho^{\gamma^{\prime}-1} \sum_{q^{\prime}} \tilde{\rho}_{q^{\prime}}^{2} \\
& -\frac{W_{0}}{2}\left(\nabla J+\nabla J_{q}\right)
\end{aligned}
$$

In the case of protons, varying the expressions (21) and (22) leads to the following expression for the Coulomb field

$$
V_{c}(\mathbf{r})=\frac{e^{2}}{2} \int d^{3} \mathbf{r}^{\prime} \frac{\rho_{p}\left(\mathbf{r}^{\prime}\right)}{\left|\mathbf{r}-\mathbf{r}^{\prime}\right|}-e^{2}\left(\frac{3}{\pi}\right)^{\frac{1}{3}} \rho_{p}^{1 / 3}(\mathbf{r}) .
$$

The particle-particle (pairing) field is

$$
\tilde{U}_{q}=\frac{t_{0}^{\prime}}{2}\left(1-x_{0}^{\prime}\right) \tilde{\rho}_{q}+\frac{t_{1}^{\prime}}{4}\left(1-x_{1}^{\prime}\right)\left[\tilde{\tau}_{q}-\frac{1}{2} \Delta \tilde{\rho}_{q}\right]+\frac{t_{3}^{\prime}}{12}\left(1-x_{3}^{\prime}\right) \rho^{\gamma^{\prime}} \tilde{\rho}_{q}
$$


Finally, the spin-orbit fields $(\propto \ell \cdot \mathbf{s})$ have the following form factors:

$$
\begin{gathered}
B_{q}=-\frac{1}{8}\left(t_{1} x_{1}+t_{2} x_{2}\right) J+\frac{1}{8}\left(t_{1}-t_{2}\right) J_{q}+W_{0} \nabla\left(\rho+\rho_{q}\right), \\
\tilde{B}_{q}=\left[\frac{t_{2}^{\prime}}{2}\left(1+x_{2}^{\prime}\right)+W_{0}^{\prime}\right] \tilde{J}_{q} .
\end{gathered}
$$

\subsection{The Hartree-Fock-Bogolyubov equations}

Writing the fields in matrix form

$$
\begin{gathered}
\mathcal{M}=\left(\begin{array}{cc}
M & \tilde{M} \\
\tilde{M} & -M
\end{array}\right), \quad \mathcal{U}=\left(\begin{array}{cc}
U-\lambda & \tilde{U} \\
\tilde{U} & -U+\lambda
\end{array}\right), \\
\mathcal{U}_{\mathrm{so}}=\left(\begin{array}{cc}
B & \tilde{B} \\
\tilde{B} & -B
\end{array}\right) \frac{j(j+1)-\ell(\ell+1)-\frac{3}{4}}{2 r} .
\end{gathered}
$$

The HFB equations read

$$
\left[-\frac{d}{d r} \mathcal{M} \frac{d}{d r}+\mathcal{U}+\mathcal{M} \frac{\ell(\ell+1)}{r^{2}}+\frac{\mathcal{M}^{\prime}}{r}+\mathcal{U}_{\mathrm{so}}\right]\left(\begin{array}{l}
u_{1} \\
u_{2}
\end{array}\right)=E\left(\begin{array}{l}
u_{1} \\
u_{2}
\end{array}\right)
$$

An $r$-dependant mixing of components and scaling described in Ref. [5] allows us to write this equation as an equation with no differential operator in the coupling terms and no first order derivative

$$
\begin{aligned}
-M^{*} \frac{d^{2}}{d r^{2}} f_{1}+V f_{1}+W f_{2} & =E f_{1} \\
M^{*} \frac{d^{2}}{d r^{2}} f_{2}-V f_{2}+W f_{1} & =E f_{2} .
\end{aligned}
$$

This last form with no first order derivative of the functions is particularly suitable for the numerical integration by the Numerov algorithm briefly discussed in section 5.2.

\subsection{Asymptotic properties of the HFB wave functions}

The asymptotic properties of the two components of the HFB quasiparticle wave functions, and their dependence on $\lambda$ and $E$, were discussed in Refs. $[17,5,18]$. Here we complement this discussion by further elements, which 
pertain mainly to weakly bound systems where the Fermi energy $\lambda$ is very small.

Assuming that $M^{*}=\frac{\hbar^{2}}{2 m}$, which anyhow is always fulfilled in the asymptotic region, and neglecting for simplicity this trivial mass factor altogether, Eqs. (34) have for large $r$ the following form

$$
\begin{aligned}
& f_{1}^{\prime \prime}+(E+\lambda) f_{1}=W f_{2} \\
& f_{2}^{\prime \prime}-(E-\lambda) f_{2}=-W f_{1} .
\end{aligned}
$$

Here we have also neglected all mean-field potentials, assuming that the distance is large enough that they are much smaller than the two characteristic constants $k^{2}=E+\lambda$ and $\kappa^{2}=E-\lambda$. Moreover, we consider the case of small Fermi energy such that there are no discrete HFB states, i.e., $E \geq-\lambda$. Within such conditions, the only remaining question is whether in the asymptotic region we can neglect the coupling potential $W$.

In order to discuss this question, we note that the coupling terms can be considered as inhomogeneities of the linear equations (35), and therefore, asymptotic solutions have the form

$$
\begin{aligned}
& f_{1}(r) \propto \sin (k r+\delta)+F_{1}(r), \\
& f_{2}(r) \propto \quad e^{-\kappa r}+F_{2}(r) .
\end{aligned}
$$

We can now iterate these solutions starting with $F_{1}(r)=F_{2}(r)=0$, which corresponds to neglecting the coupling terms in the zero order. Then, in the first order we see that in the equation for $f_{1}$ the coupling term can still be neglected as compared to the large term $(E+\lambda) f_{1}$. Therefore, to all orders we have $F_{1}(r)=0$, and the asymptotic solution $f_{1}(r) \propto \sin (k r+\delta)$ defines the inhomogeneity $W f_{1}$ of the equation for $f_{2}$.

We arrive here at the conclusion that the asymptotic form of $f_{2}$ may involve two terms and a more detailed analysis is needed before concluding which one dominates. To this end, we note that the coupling potential $W$ depends on the sum of products of lower and upper components, and therefore has a general form of

$$
W(r) \propto W_{\text {osc }}(r) e^{-\mu r} / r^{2}
$$

where $W_{\text {osc }}(r)$ is an oscillating function of order $1, \mu$ is the decay constant, which may characterize another quasiparticle state than the one with energy $E$, and the factor $r^{2}$ comes from the volume element, cf. Eq. (10). We see here that the term $F_{2}(r)$ in the asymptotic form of $f_{2}(r)$ vanishes as $e^{-\mu r}$, but it is difficult to say which one of the two terms dominates asymptotically. We only note that for small quasiparticle energies the decay constant $\kappa$ is also small and hence the first term is then more likely to dominate. 

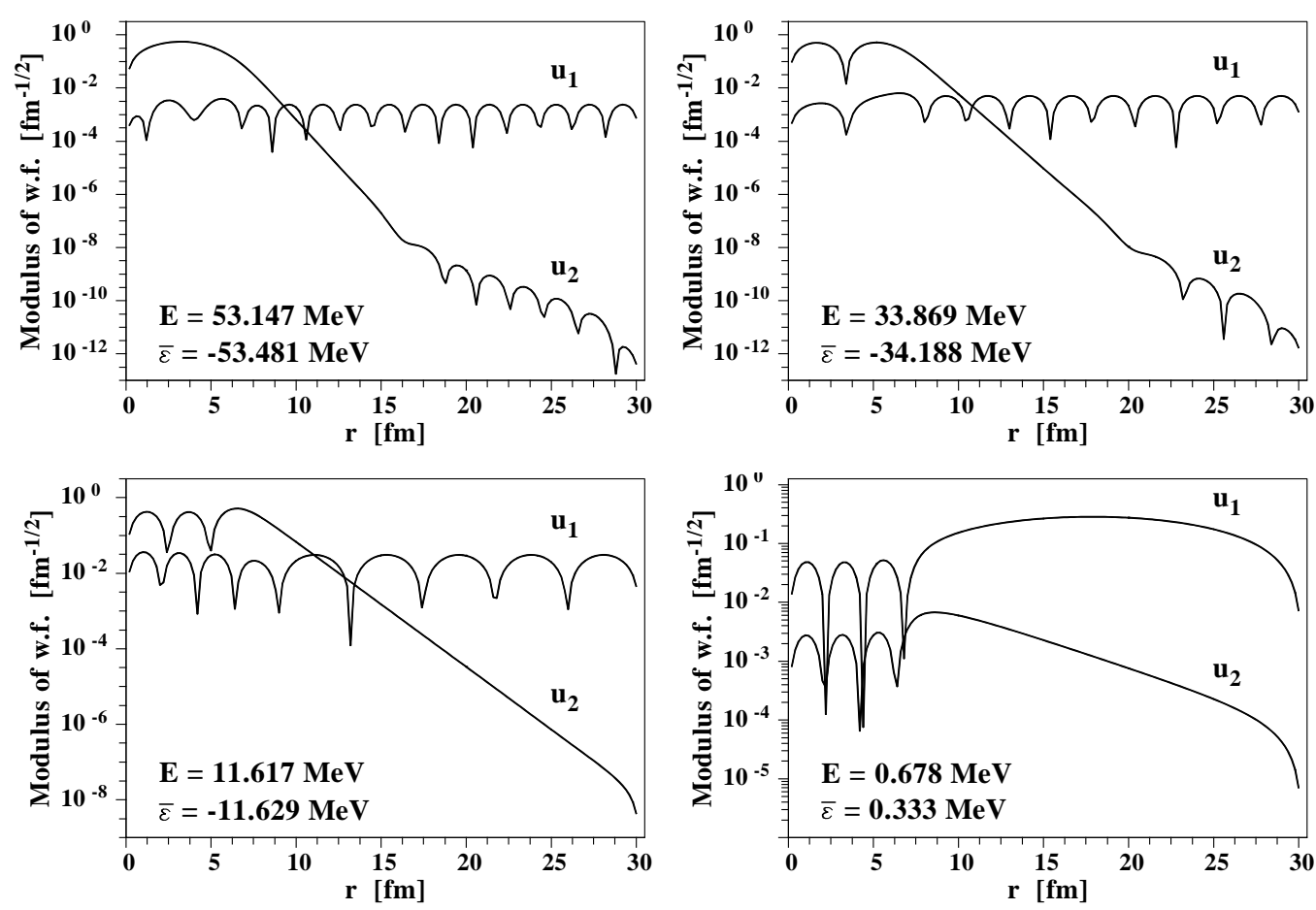

Fig. 1. HFB quasiparticle wave functions $u_{1}(r)$ and $u_{2}(r)$ of the four lowest states in ${ }^{174} \mathrm{Sn}$.

In order to illustrate the above discussion, we have performed the HFB calculations in ${ }^{174} \mathrm{Sn}$, where $\lambda_{n}=-0.345 \mathrm{MeV}$ and the lowest $\ell=0$ quasiparticle state of $E=0.429 \mathrm{MeV}$ (for $R_{\mathrm{box}}=30 \mathrm{fm}$ ) leads to a very diffused coupling potential with small decay constant $\mu$. Quasiparticle wave functions corresponding to the four lowest $\ell=0$ quasiparticle states are shown in Fig. 1. One can see that the asymptotic forms of the second components $u_{2}(r)$ of the two lowest quasiparticle states are not affected by the second term $F_{2}(r)$, at least up to $30 \mathrm{fm}$. Only the third and fourth states switch at large distances to the oscillating asymptotic forms with a smaller decay constant. This happens at rather large distances where the densities are anyhow very small. Hence the change in the asymptotic properties does not affect any important nuclear observables.

In all cases that we have studied, the practical importance of the second term $F_{2}(r)$ is negligible. However, its presence precludes simple analytic continuation of the wave functions in the asymptotic region. We would like to stress that the asymptotic forms discussed in this section are numerically stable, and that they are unrelated to the numerical instabilities discussed in Sec. 5.3 below. 


\subsection{Pairing correlations and divergence of the energy}

The use of a zero range interaction in the particle-particle channel leads to the energy divergence problem $[9,19]$ related to the fact that the short-range behaviour of the abnormal density is

$$
\tilde{\rho}(r)=\lim _{r^{\prime} \rightarrow r} \tilde{\rho}\left(r, r^{\prime}\right) \propto \frac{1}{\left|r-r^{\prime}\right|} .
$$

The other abnormal densities diverge as well, but along that the pairing correlations also induce the divergence of the kinetic density $\tau(r)$. One simple way to overcome the divergence of the energy is to introduce a cut-off energy $E_{\text {cut }}$ in the summations of all the densities

$$
\sum_{E_{i}>0} \rightarrow \sum_{0<E_{i}<E_{\mathrm{cut}}} .
$$

This cut-off somehow simulates the finite range of the interaction in the pairing channel. It therefore constitutes an additional parameter of the interaction and must be adjusted along with the other quantities which define the pairing interaction, see e.g. Ref. [18].

A more elegant way to prevent the divergence was recently proposed in Refs. $[9,19]$. It is based on the subtractions of the divergent part of the abnormal density. In its present implementation in the code HFBRAD, this method can only be applied if the pairing force does not contains non-local terms (i.e. gradient terms). In that case, the pairing energy depends only on $\tilde{\rho}$ (and possibly on $\rho$ but not on $\tilde{\tau}$ or $\tilde{J})$, i.e.,

$$
\mathcal{E}_{\text {pair }}=\sum_{q}\left[\frac{t_{0}^{\prime}}{4}\left(1-x_{0}^{\prime}\right)+\frac{t_{3}^{\prime}}{24}\left(1-x_{3}^{\prime}\right) \rho^{\gamma^{\prime}}\right] \tilde{\rho}_{q}^{2}=\sum_{q} g[\rho] \tilde{\rho}_{q}^{2},
$$

and the pairing potential depends linearly on $\tilde{\rho}$,

$$
\tilde{U}_{q}=2 g[\rho] \tilde{\rho}_{q} .
$$

The regularized pairing field can then be written as

$$
\tilde{U}_{q}^{\mathrm{reg}}=2 g[\rho] \tilde{\rho}_{q}^{\mathrm{reg}} \equiv 2 g_{\mathrm{eff}}[\rho] \tilde{\rho}_{q} .
$$

Calculation of the various densities is performed by summing up the quasiparticle contributions up to a maximum energy $E_{\max }$, but because of the regularization, this maximum energy has not the meaning of a cut-off energy $E_{\text {cut }}$.

It can be shown [19] that when we evaluate the total energy of the system, 
the pairing energy must be defined as

$$
\mathcal{E}_{\text {pair }}=\sum_{q} g_{\mathrm{eff}}[\rho] \tilde{\rho}_{q}^{2}
$$

With this definition, the kinetic and pairing energies both diverge but the sum, and indeed the total energy, does not [19]. If the pairing energy density involves kinetic or gradient terms (like it was implemented for the Skyrme interaction $\mathrm{SkP}$ [5]), the regularization would require the subtraction of higher order terms. This possibility in not implemented in the present version of the code. Finally, let us note that relations (21)-(26) in Ref. [19] imply that the variation of quantities $k_{F}$ and $k_{c}$ with respect to the densities should be included in the mean fields. This contribution is supposed to be small and is not taken into account in the code. On the other hand, for densitydependent pairing interaction, the variation with respect to the explicit density dependence is taken into account in the code, and gives a contribution to the energy of the order of a few $\mathrm{keV}$.

\section{The effective Skyrme force}

For a general overview of the foundations and properties of the Skyrme force we refer the reader to the review article [20] and references therein. The Skyrme force is an effective interaction depending on a limited number of parameters,

$$
\begin{aligned}
V_{12}= & t_{0}\left(1+x_{0} P_{\sigma}\right) \delta+\frac{1}{2} t_{1}\left(1+x_{1} P_{\sigma}\right)\left(\mathbf{k}^{\prime 2} \delta+\delta \mathbf{k}^{2}\right)+t_{2}\left(1+x_{2} P_{\sigma}\right) \mathbf{k}^{\prime} \cdot \delta \mathbf{k} \\
& +\frac{1}{6} t_{3}\left(1+x_{3} P_{\sigma}\right) \rho^{\gamma} \delta+\mathrm{i} W_{0}\left(\boldsymbol{\sigma}_{1}+\boldsymbol{\sigma}_{2}\right) \cdot\left(\mathbf{k}^{\prime} \times \delta \mathbf{k}\right),
\end{aligned}
$$

where $\delta$ is a short notation for $\delta\left(\mathbf{r}_{1}-\mathbf{r}_{2}\right), \mathbf{k}=\mathbf{k}_{1}-\mathbf{k}_{2}$ acting on the right and $\mathbf{k}^{\prime}=\mathbf{k}_{1}-\mathbf{k}_{2}$ acting on the left.

The parameters of the Skyrme forces were fitted in the literature to reproduce various bulk nuclear properties as well as selected properties of some nuclei (usually doubly magic nuclei). Simplifications have often been made in the expression of the functional (19), like treatment of the Coulomb exchange term with the Slater approximation and/or omission of the two-body center of mass contribution or of the " $J^{2}$ " terms. The latter corresponds to the fourth line in Eq. (19) and to the two first terms in the spin-orbit mean field, Eq. (29). It is important to keep in mind that one should use each parametrization of the functional within the same simplifications with which it has been adjusted to data.

For all forces implemented in HFBRAD, the force in the particle-particle channel 
is chosen to be

$$
V_{12}^{\prime}=\left(t_{0}^{\prime}+\frac{t_{3}^{\prime}}{6} \rho^{\gamma^{\prime}}\right) \delta
$$

Table 1 gives the different sets of parameters for the force in the particle-hole channel, while table 2 gives the parameters in the particle-particle channel. It is important to keep in mind that these latter parameters have been adjusted along with a given cut-off and cut-off diffuseness, which are two additional parameters of the force.

Table 1

\begin{tabular}{|c|rrrrc|}
\cline { 2 - 6 } \multicolumn{1}{c|}{} & SIII $[4]$ & SkM $^{*}[21]$ & SLy4 $[22]$ & SLy5 $[22]$ & SkP [5] \\
\hline$t_{0}$ & -1128.75 & -2645.0 & -2488.913 & -2488.345 & -2931.6960 \\
$t_{1}$ & 395.0 & 410.0 & 486.818 & 484.230 & 320.6182 \\
$t_{2}$ & -95.0 & -135.0 & -546.395 & -556.690 & -337.4091 \\
$t_{3}$ & 14000.0 & 15595.0 & 13777.0 & 13757.0 & 18708.96 \\
$x_{0}$ & 0.45 & 0.09 & 0.834 & 0.776 & 0.2921515 \\
$x_{1}$ & 0.0 & 0.0 & -0.344 & -0.317 & 0.6531765 \\
$x_{2}$ & 0.0 & 0.0 & -1.000 & -1.000 & -0.5373230 \\
$x_{3}$ & 1.0 & 0.0 & 1.354 & 1.263 & 0.1810269 \\
$\gamma$ & 1.0 & $1 / 6$ & $1 / 6$ & $1 / 6$ & $1 / 6$ \\
$W_{0}$ & 130.0 & 120.0 & 123.0 & 125.00 & 100.000 \\
\hline$J^{2}$ & No & No & No & Yes & Yes \\
\hline
\end{tabular}

Parameters in the particle-hole channel for the different versions of the Skyrme forces implemented in the code HFBRAD. The last line indicates if the $J^{2}$ terms are included in the Skyrme functional when the force is used.

Three kinds of pairing forces have been adjusted: (i) the volume pairing (the pairing field follows the shape of the density), below denoted by " $\rho$ ", (ii) the surface pairing (the pairing field is peaked at the surface and follows roughly the variations of the density), below denoted by " $\delta \rho$ ", and (iii) the mixed pairing (a compromise between the two first two), below denoted by " $\rho+\delta \rho$ ". All pairing parameters have been adjusted in order to give a mean neutron gap of $1.245 \mathrm{MeV}$ in ${ }^{120} \mathrm{Sn}$. 


\begin{tabular}{|l|rrr|rrr|}
\cline { 2 - 7 } \multicolumn{1}{c|}{} & \multicolumn{3}{c|}{ cut-off } & \multicolumn{3}{c|}{ regularization } \\
\cline { 2 - 7 } \multicolumn{1}{c|}{} & $\rho$ & $\delta \rho$ & $\rho+\delta \rho$ & $\rho^{r}$ & $\delta \rho^{r}$ & $(\rho+\delta \rho)^{r}$ \\
\hline$t_{0}^{\prime}(\mathrm{SIII})$ & -159.6 & -483.2 & -248.5 & -197.6 & -807.0 & -316.9 \\
\hline$t_{0}^{\prime}\left(\mathrm{SkM}^{*}\right)$ & -148.6 & -452.6 & -233.9 & -184.7 & -798.4 & -300.7 \\
\hline$t_{0}^{\prime}(\mathrm{SLy} 4)$ & -186.5 & -509.6 & -283.3 & -233.0 & -914.2 & -370.2 \\
\hline$t_{0}^{\prime}(\mathrm{SLy} 5)$ & -179.9 & -504.9 & -275.8 & -222.7 & -901.9 & -356.6 \\
\hline$t_{0}^{\prime}(\mathrm{SkP})$ & -131.6 & -429.5 & -213.1 & -196.6 & -1023.0 & -326.5 \\
\hline$t_{3}^{\prime}$ & 0 & $-37.5 t_{0}^{\prime}$ & $-18.75 t_{0}^{\prime}$ & 0 & $-37.5 t_{0}^{\prime}$ & $-18.75 t_{0}^{\prime}$ \\
\hline$\gamma^{\prime}$ & 1 & 1 & 1 & 1 & 1 & 1 \\
\hline
\end{tabular}

Table 2

Parameters in the particle-particle channel for the different versions of the Skyrme forces implemented in the code HFBRAD. The three first columns corresponds to the use of a cut-off at $60 \mathrm{MeV}$ with a Fermi shape and a diffuseness of $1 \mathrm{MeV}$, the three columns on the right corresponds to the regularization procedure of the pairing field described in Sec. 2.7.

\section{Observables and single particle properties}

\subsection{Hartree-Fock equivalent energies, radii, and nodes of quasiparticle wave functions}

For each quasiparticle state, in addition to its quasiparticle energy $E_{i}$ given by the HFB equation, one has several other characteristics, which are defined the following way:

- the occupation factor $N_{i}$ which is the norm of the lower component of the HFB quasiparticle wave function;

- the (Hartree-Fock) equivalent energy $\bar{\varepsilon}_{i}$ which is defined by applying the BCS type formula to the occupation factor $N_{i}$, i.e.,

$$
N_{i}=\frac{1}{2}\left(1-\frac{\bar{\varepsilon}_{i}-\lambda}{E_{i}}\right),
$$

which gives

$$
\bar{\varepsilon}_{i}=\lambda+E_{i}\left(2 N_{i}-1\right) ;
$$

- in the same spirit, the equivalent gap $\bar{\Delta}_{i}$ is defined by applying the BCS type formula to the HFB quasiparticle energy $E_{i}$, i.e.,

$$
E_{i}=\sqrt{\left(\bar{\varepsilon}_{i}-\lambda\right)^{2}+\bar{\Delta}_{i}^{2}}
$$


which gives

$$
\Delta_{i}=2 E_{i} \sqrt{N_{i}\left(1-N_{i}\right)}
$$

- the rms radius $\sqrt{\left\langle r_{i}^{2}\right\rangle}$ is calculated using the lower component of the quasiparticle wave function

$$
\left\langle r_{i}^{2}\right\rangle=\int_{0}^{R_{\mathrm{box}}} \mathbf{r}^{2} \varphi_{2, i}^{2}(\mathbf{r}) d^{3} \mathbf{r}
$$

- the number of nodes of the HFB quasiparticle wave function is defined as the number of nodes (including the node at the origin but not the one at infinity) of the component which has the greatest amplitude.

These characteristics of the quasiparticle states can be found in the output file hfb_n_p.spe, see Sec. 7.

\subsection{Canonical basis}

The canonical states are obtained by diagonalizing the density matrix (see e.g. Refs. $[10,18]$ for the interpretation of the canonical basis)

$$
\int d^{3} \mathbf{r}^{\prime} \rho\left(\mathbf{r}, \mathbf{r}^{\prime}\right) \psi_{i}\left(\mathbf{r}^{\prime}\right)=v_{i}^{2} \psi_{i}(\mathbf{r})
$$

As discussed in Refs. [5,18], all canonical states have localized wave functions and form a basis. The energies of the canonical states are defined as the diagonal matrix elements of the Hartree-Fock field $h$ in the canonical basis,

$$
\varepsilon_{i}=\left\langle\psi_{i}|h| \psi_{i}\right\rangle
$$

and the pairing gaps associated with these states are the diagonal matrix elements of the pairing field,

$$
\Delta_{i}=\left\langle\psi_{i}|\tilde{h}| \psi_{i}\right\rangle
$$

Finally, the canonical quasiparticle energy can be defined using the same kind of formula as (48),

$$
E_{i}^{\mathrm{can}}=\sqrt{\left(\varepsilon_{i}-\lambda\right)^{2}+\Delta_{i}^{2}}
$$

while the occupation probabilities of canonical states are given by

$$
v_{i}^{2}=\frac{1}{2}\left(1-\frac{\varepsilon_{i}-\lambda}{E_{i}^{\mathrm{can}}}\right),
$$

These characteristics of the canonical states can be found in the output file hfb_n_p.spe, see Sec. 7 . 


\subsection{Observables and other characteristic quantities of the system}

The rms radii for protons and neutrons are defined as $(q=n$ or $p)$

$$
\left\langle r_{q}^{2}\right\rangle=\int_{0}^{R_{\mathrm{box}}} \mathbf{r}^{2} \rho_{q}^{2}(\mathbf{r}) d^{3} \mathbf{r} .
$$

The charge radius is obtained from the proton radius by taking into account the proton charge distribution in an approximate way, i.e.,

$$
\left\langle r_{\mathrm{ch}}^{2}\right\rangle=\left\langle r_{p}^{2}\right\rangle+\langle r\rangle_{P}^{2} .
$$

with $\langle r\rangle_{P}=0.8 \mathrm{fm}$. The mean gaps are the average values of the pairing fields

$$
\left\langle\Delta_{q}\right\rangle=-\frac{\operatorname{Tr} \tilde{h}_{q} \rho}{A} .
$$

The fluctuations of the particle numbers are defined as $\left\langle\hat{N}_{q}^{2}-\left\langle\hat{N}_{q}\right\rangle^{2}\right\rangle$ and given by $2 \operatorname{Tr}\left[\rho_{q}^{2}-\rho_{q}\right]$.

Finally, the rearrangement energy, which comes from the density dependence of the force, and which shows how much the force is modified by the medium effects, is given by

$$
\begin{aligned}
E_{\text {rear }} & =\frac{t_{3}}{24} \gamma \rho^{\gamma}\left[\left(1+\frac{x_{3}}{2}\right) \rho^{2}-\left(x_{3}+\frac{1}{2}\right) \sum_{q} \rho_{q}^{2}\right] \\
& +\frac{t_{3}^{\prime}}{48} \gamma^{\prime}\left(1-x_{3}^{\prime}\right) \rho^{\gamma^{\prime}} \sum_{q} \tilde{\rho}_{q}^{2}-\frac{e^{2}}{4}\left(\frac{3}{\pi}\right)^{\frac{1}{3}} \rho_{p}^{4 / 3} .
\end{aligned}
$$

\section{$5 \quad$ Numerical treatment of the problem}

\subsection{The problem inside a box}

The system of differential equations (34) is solved in coordinate representation in a spherical box for a given choice of boundary conditions. Here we briefly introduce notations that useful for the discussion that follows and discuss the validity of approximations to solve the differential problem. The radial coordinate $r$ is approximated by a mesh of points spaced by the step $h: r_{n}=$ $n h$, with $n \in\{0,1, \ldots, N\}$. For any quantity $F(r)$ approximated on the mesh we use notation $F_{n} \equiv F\left(r_{n}\right)$. 
On the wall of the box, we use two possible boundary conditions: vanishing wave functions (Dirichlet condition) or vanishing derivatives of the wave functions (Neumann condition). With this choice, the continuous part of the spectrum is discretized. Important questions are how well the continuum is approximated by this discretization and whether it does not introduce numerical artifacts when solving system of equations (34). A discussion concerning the behaviour of the resonant like solution inside the box can be found in [5]. The non-linearity of the equations makes it difficult to discuss further the effect of the discretization of the spectrum, nevertheless, the numerical examples shown in Sec. 8 show that such numerical method provides a fairly good approximation of the exact problem. In particular, it can be observed that once a sufficiently large box is used, the global properties of the nucleus become perfectly independent of its size.

\subsection{The Numerov algorithm for the HFB equation}

The Numerov (Cowell) method [23] is the standard technique for a precise integration of second-order differential equations; here we only give some details pertaining to its application to the system of two HFB equations (34). Let function $y(r)$ be the solution of the differential equation

$$
y^{\prime \prime}=F(y, r) \text {. }
$$

The Numerov algorithm is based on the finite difference formula for three consecutive points on a mesh with step $h$,

$$
y_{n+1}-2 y_{n}+y_{n-1}=\frac{h^{2}}{12}\left(y_{n-1}^{\prime \prime}+10 y_{n}^{\prime \prime}+y_{n+1}^{\prime \prime}\right)+o\left(h^{6}\right)
$$

where $o\left(h^{6}\right)$ means that the error in this relation is of the order $h^{6}$. Combining this formula with (60), one obtains the relation

$$
\left(1-\frac{h^{2}}{12} F_{n+1}\right) y_{n+1}-2\left(1+\frac{5 h^{2}}{12} F_{n}\right) y_{n}+\left(1-\frac{h^{2}}{12} F_{n-1}\right) y_{n-1}=0
$$

which is obviously also valid if $y$ is a vector and $F$ a square matrix. Introducing notations

$$
A_{n}=\left(1-\frac{h^{2}}{12} F_{n}\right)
$$

and

$$
G_{n}=A_{n} y_{n}
$$


one obtains the recurrence relation (Numerov iterations),

$$
\left\{\begin{array}{l}
G_{n+1}=12 y_{n}-10 G_{n}-G_{n-1} \\
y_{n+1}=A_{n+1}^{-1} G_{n+1}
\end{array}\right.
$$

In the present applications, $A_{n}$ is a $2 \times 2$ matrix (position and energy dependent) and the calculation of its inverse could be the bottleneck of the procedure if it is not computed cleverly. This can be done in the following way. Using the notations introduced in Sec. 2.5, one can write matrix $A_{n}$ in an explicit form as

$$
A_{n}=\left(\begin{array}{cc}
1-\frac{h^{2}}{12} \frac{V_{n}}{M_{n}^{*}}+\frac{h^{2}}{12} \frac{E}{M_{n}^{*}} & -\frac{h^{2}}{12} \frac{W_{n}}{M_{n}^{*}} \\
\frac{h^{2}}{12} \frac{W_{n}}{M_{n}^{*}} & 1-\frac{h^{2}}{12} \frac{V_{n}}{M_{n}^{*}}-\frac{h^{2}}{12} \frac{E}{M_{n}^{*}}
\end{array}\right),
$$

whereby its inverse is given by

$$
\begin{aligned}
A_{n}^{-1}=\frac{1}{\left(1-\frac{h^{2} V_{n}}{12 M_{n}^{*}}\right)^{2}+\left(\frac{h^{2} W_{n}}{12 M_{n}^{*}}\right)^{2}-E^{2}\left(\frac{h^{2}}{12 M_{n}^{*}}\right)^{2}} & \left(\begin{array}{cc}
1-\frac{h^{2}}{12} \frac{V_{n}}{M_{n}^{*}}-\frac{h^{2}}{12} \frac{E}{M_{n}^{*}} & \frac{h^{2}}{12} \frac{W_{n}}{M_{n}^{*}} \\
-\frac{h^{2}}{12} \frac{W_{n}}{M_{n}^{*}} & 1-\frac{h^{2}}{12} \frac{V_{n}}{M_{n}^{*}}+\frac{h^{2}}{12} \frac{E}{M_{n}^{*}}
\end{array}\right) .
\end{aligned}
$$

It appears clearly that a lot of time can be saved by calculating and storing the energy independent terms,

$$
1-\frac{h^{2} V_{n}}{12 M_{n}^{*}}, \frac{h^{2}}{12 M_{n}^{*}}, \frac{h^{2} W_{n}}{12 M_{n}^{*}} \text { and }\left(1-\frac{h^{2} V_{n}}{12 M_{n}^{*}}\right)^{2}+\left(\frac{h^{2} W_{n}}{12 M_{n}^{*}}\right)^{2},
$$

only once in each $(\ell j q)$-block; then the energy-dependent terms can be in each iteration calculated very rapidly.

The eigen energies are found by integrating two linearly independent solutions, $y^{(1+)}(r)$ and $y^{(2+)}(r)$, from the origin to a given point $r_{m}$, called the matching point. These two solutions are selected by imposing that either the first or the second component of the quasiparticle wave function vanishes near the origin, i.e.,

$$
y^{(1+)}(r) \sim\left(\begin{array}{c}
r^{\ell+1} \\
0
\end{array}\right), \quad y^{(2+)}(r) \sim\left(\begin{array}{c}
0 \\
r^{\ell+1}
\end{array}\right), \quad \text { for } r \rightarrow 0,
$$

whereby we have

$$
y_{0}^{(1+)}=\left(\begin{array}{l}
0 \\
0
\end{array}\right), \quad y_{0}^{(2+)}=\left(\begin{array}{l}
0 \\
0
\end{array}\right)
$$


and

$$
y_{1}^{(1+)}=\left(\begin{array}{c}
A h^{\ell+1} \\
0
\end{array}\right), \quad y_{1}^{(2+)}=\left(\begin{array}{c}
0 \\
B h^{\ell+1}
\end{array}\right) \text {. }
$$

Next, another two linearly independent solutions, $y^{(1-)}(r)$ and $y^{(2-)}(r)$, are found by the backward integration from the wall of the box $R_{\text {box }} \equiv r_{N}$ to $r_{m}$, again imposing that either the first or the second component of the quasiparticle wave function vanishes near $r_{N}$ for the Dirichlet boundary conditions, i.e.,

$$
y^{(1-)} \sim\left(\begin{array}{c}
r-r_{N} \\
0
\end{array}\right), \quad y^{(2-)} \sim\left(\begin{array}{c}
0 \\
r-r_{N}
\end{array}\right), \quad \text { for } r \rightarrow r_{N},
$$

whereby we have

$$
y_{N}^{(1-)}=\left(\begin{array}{l}
0 \\
0
\end{array}\right), \quad y_{N}^{(2-)}=\left(\begin{array}{l}
0 \\
0
\end{array}\right)
$$

and

$$
y_{N-1}^{(1-)}=\left(\begin{array}{c}
C h \\
0
\end{array}\right), \quad y_{N-1}^{(2-)}=\left(\begin{array}{c}
0 \\
D h
\end{array}\right) .
$$

For the Neumann boundary conditions, at the left-hand-sides of Eqs. (71)(73) one replaces functions by derivatives. Finally, one obtains four solutions valid everywhere except at the matching point $r_{m}$ and depending on the four constants $A, B, C$, and $D$.

The boundary conditions (69)-(70) and (72)-(73) determine the initial values $\left(G_{0}, G_{1}\right)$ and $\left(G_{N}, G_{N-1}\right)$, respectively, needed to start the Numerov iterations of (65). Obviously, a correct limit should be taken when calculating values of $G_{0}$ from Eq. (64). It turns out that one obtains $G_{0}=0$ for all partial waves except $\ell=1$, which is the case that deserves a little more attention. For example, focusing our attention on one of the solutions,

$$
y^{(1+)}(r)=\left(\begin{array}{c}
A r^{2} \\
0
\end{array}\right)
$$

we use the fact that in the vicinity of the origin $A(r)$ is dominated by the centrifugal term, so that its diagonal matrix elements are close to $-\frac{h^{2}}{12} \frac{2}{r^{2}}$. This leads to the result

$$
G_{0}^{(1+)}=\lim _{r \rightarrow 0} A(r) y(r)=-\frac{A h^{2}}{6}\left(\begin{array}{l}
1 \\
0
\end{array}\right) .
$$

An analogous result can be obtained for the other solution at the origin. 
The eigenenergies are found by requiring that the full solution $y=A y_{1}^{(+)}+$ $B y_{2}^{(+)}=C y_{1}^{(-)}+D y_{2}^{(-)}$and its first derivative are continuous at $r=r_{m}$ (see for example [24]). This matching conditions read

$$
\left(\begin{array}{cccc}
y_{m}^{(1+)} & y_{m}^{(2+)} & -y_{m}^{(1-)} & -y_{m}^{(2-)} \\
y_{m}^{\prime(1+)} & y_{m}^{\prime(2+)} & -y_{m}^{\prime(1-)} & -y_{m}^{(2-)}
\end{array}\right)\left(\begin{array}{l}
A \\
B \\
C \\
D
\end{array}\right)=M_{4}\left(\begin{array}{l}
A \\
B \\
C \\
D
\end{array}\right)=0 .
$$

The HFB quasiparticle wave functions having two components, the matching conditions amount to searching for zeros of the determinant of the $4 \times 4$ matrix $M_{4}$ in function of the quasiparticle energy. For $\ell=0$, the matching point $r_{m}$ is chosen to be near the point where the central part of the Hartree-Fock potential is half of its maximum, while for increasing values of $\ell$ it is gradually shifted to the exterior. A systematic search allows to find the intervals where the zeros of this determinant are located, and then the Newton method is used to find their exact locations to an arbitrary precision.

For vanishing determinant, Eq. (76) is used to determine three of the multiplicative constants in terms of the fourth one. This is done by extracting a $3 \times 3$ matrix $M_{3}$ out of $M_{4}$ and inverting it. Among the four possible choices one may have for $M_{3}$ we retain the one for which the product $M_{3} \times M_{3}^{-1}$ is closest to unity. Finally, the fourth multiplicative constant is fixed by the normalization condition of the wave function.

When approaching the self-consistent solution, the eigenenergies do not change very much between two consecutive Bogolyubov iterations. We take advantage of this fact by keeping in memory the intervals where the solutions are located and using them as initial guesses for the next iteration. This results in finding solutions for eigenenergies faster and faster as we get closer to the converged self-consistent state.

\section{$5.3 \quad$ Numerical instabilities}

If we try to solve the HFB equations in rather big boxes (typically beyond 30 or $40 \mathrm{fm}$ depending on the mass of the nucleus) we encounter strong numerical instabilities. This problem is not trivially related to the accumulation of errors during the Numerov iteration, because it disappears if the nucleus is treated within the HF approximation instead of HFB. The origin of the problem is in the fact that at large distances the upper and lower HFB wave functions differ by many orders of magnitude, and thus calculations within any number of significant digits must fail at some distance, especially if the small component 
decreases very fast exponentially. This is so because the small component becomes easily polluted by the large component even if they are coupled by very small matrix elements.

This numerical instability can be removed by neglecting the pairing field beyond a certain large distance $R_{\text {cut }}$. Instead of the full pairing field we consider the truncated field given by

$$
\Delta_{\text {cut }}(r)= \begin{cases}\Delta(r) & \text { for } r \leq R_{\text {cut }} \\ 0 & \text { for } r>R_{\text {cut }}\end{cases}
$$

It will be shown in section 8.1 that this truncation has no significant effects on the solution if $R_{\text {cut }}$ is far enough from the origin. Note that by removing the coupling between the lower and upper components one also removes the second term in the asymptotic form of the lower component, see Eq. (36).

Another source of numerical instabilities may come from an accidental degeneracy of deep hole and particle states. In the HFB approach the deep hole states are embedded in the continuum of particle states. The deep hole states are extremely narrow and thus insensitive to the size of the integration box. On the other hand, the discretized particle states are driven by the boundary condition and their spectrum becomes denser as the box is enlarged. As a result, a deep hole state and a quasiparticle (particle like) state can become quasi degenerate and the systematic search for the solution [corresponding to the matching condition (76)] can miss both of them simultaneously. This situation can show up during the iterations and disappear at the final solution; in such a case this numerical accident has no consequence on the final solution. If the quasi degeneracy is met close to the final self-consistent solution, the iterations may not converge. This can be overcome by using a smaller energy step or by slightly changing the size of the integration box in order to lift the degeneracy.

\section{Input data file}

The code HFBRAD is driven using a single input file which contains all information on the geometry of the system, parameters of the force, demanded output and nuclei to be computed. The data are read in namelist, which is a Fortran-90/95 feature. All variables in a namelist are optional and acquire a default value when absent. 


\subsection{The namelist input}

The namelist input contains the variables summarized on table 3 with their default values. The variables are

- force: sets the parameters of the force in the particle-hole channel, possible values are SIII, SKM*, SKP, SLY4 and SLY5, it must be written in uppercase.

- mesh_points: number of mesh points in the box minus one since integration is made from $i=0$ to $i=$ mesh_points.

- integ_step: distance between two points on the mesh, in fermis.

- itmax: maximum number of iterations to obtain the convergence of the mean fields and total energy.

- bogolyubov (2): logical variables to choose between the HF or HFB approximation, the first element is for neutrons and the second one for protons.

- eps_energy: maximum relative variation on the total energy before the iterations stops.

- max_delta: in addition to the previous convergence condition, the maximum variation on the sum of the neutron and proton pairing gaps must be smaller than this value.

- regularization: logical variable used to switch on or off the regularization of the abnormal density.

- pairing_force: integer used to choose the pairing interaction between: full Skyrme force (0), volume pairing (1), surface pairing (2) or mixed pairing (3).

- boundary_condition: integer, Dirichlet condition at the box radius (0), Neumann (1), Dirichlet for even $\ell$ and Neumann for odd $\ell$ (2), or Neumann for even $\ell$ and Dirichlet for odd $\ell$ (3).

- xmu: part of the field of the previous iteration which is kept to build the field for the next iteration.

\subsection{The namelist nucleus}

This namelist describes the nucleus to be computed, it can be repeated as many times as needed in the input file. Along with the neutron and proton numbers, other quantities can be set, notably the parameters of the Skyrme force in the particle-particle channel. If a variable is not present in the list and has not been present in the previous namelists nucleus, it acquires a default value (see table 4). If a variable is not present but was present in a previous namelist, it keeps its previous value. The variables are 


\begin{tabular}{|l|c|r|}
\hline Variable & type & Default value \\
\hline force & character*4 & SLY4 \\
mesh_points & integer & 80 \\
integ_step & real & 0.25 \\
itmax & integer & 680 \\
bogolyubov(2) & logical & $(/ \mathrm{T}, \mathrm{T} /)$ \\
eps_energy & real & $1 . \mathrm{e}-8$ \\
max_delta & real & $5 . \mathrm{e}-7$ \\
regularization & logical & $\mathrm{F}$ \\
pairing_force & integer & 1 \\
boundary_condition & integer & 0 \\
xmu & real & 0.8 \\
\hline
\end{tabular}

Table 3

Variables from the namelist input. The type real means real of the kind chosen in the module in cste. $f 90$.

- neutron and proton: number of neutrons and protons for the nucleus. If one of this number is negative then the program will let vary the number of particles in order to reach the corresponding drip line.

- j_max (2): maximum values of $2 J$ (first for neutrons, second for protons). It is reasonable to use different values for neutrons and protons in the case of very asymmetric or semi magic nuclei.

- cut_off and cut_diffuseness: If the regularization procedure is used, the densities are built by summing up contributions from the quasiparticle states up to the maximum equivalent energy (47) $E_{\max }$ given by cut_off, if not, the summation is made up to the cut-off equivalent energy $E_{\text {cut }}(47)$ given by cut_off with a smooth Fermi profile of diffuseness cut_diffuseness, see Sec. 2.7.

- $r_{-}$cut: value of distance $R_{\text {cut }}$ beyond which the paring fields are neglected when integrating the HFB equations, see Eq. (77).

- e_step: initial step in energy (in $\mathrm{MeV}$ ) for the search of the solution of the HFB equations.

- skt0p and skt3p: parameters of the force in the particle-particle channel as defined in equation (45).

- read_pot: This string can be used to give a name of a file where the initial potentials are read. The number of mesh points can be different in the run and in the saved potentials but the integration step must be the same.

- densities, meanfields, quasiparticle and canonical_states: logical flags which can be set if one wants to save the densities, mean fields and quasiparticle and canonical states wave functions at the end of the run. 
Table 4

\begin{tabular}{|l|c|r|}
\hline Variable & type & Default value \\
\hline neutron & integer & - \\
proton & integer & - \\
j_max $(2)$ & integer & $(/ 21,21 /)$ \\
cut_off & real & 60.0 \\
cut_diffuseness & real & 1.0 \\
r_cut & real & 30.0 \\
e_step & real & calculated \\
skt0p & real & predefined \\
skt3p & real & predefined \\
read_pot & character*40 & "'” \\
densities & logical & F \\
meanfields & logical & F \\
quasiparticles & logical & F \\
canonical_states & logical & F \\
\hline
\end{tabular}

Variables from the namelist nucleus. The neutron and proton numbers have no default values. If the energy step is not present in the list it is calculated as a function of the box radius. By default, the values of sktop and skt3p are fixed by the kind of pairing force chosen in the namelist input.

\section{Output files}

In addition to the optional files containing the densities, mean fields and wave functions, at the end of the run the following output files are saved:

- $h f b \_n \_p . s p e:$ where $\mathrm{n}$ and $\mathrm{p}$ are the neutron and proton numbers. This file contains the quasiparticle spectrum, the canonical states spectrum (if the flag canonical_states was set) and the mean fields.

- hfb.summary: this files summarizes the global properties for all the nuclei in the run. 
Table 5

\begin{tabular}{|l|cccc|}
\hline Boundary condition & $E$ & $\left\langle\Delta_{N}\right\rangle$ & $\lambda_{N}$ & $\sqrt{\left\langle r_{N}^{2}\right\rangle}$ \\
\hline Dirichlet & -1131.863 & -1.431 & -1.067 & 5.264 \\
Neumann & -1131.862 & -1.431 & -1.067 & 5.264 \\
\hline
\end{tabular}

Total binding energy, mean pairing gap, Fermi energy (all in $\mathrm{MeV}$ ) and neutron rms radius (in $\mathrm{fm}$ ) in ${ }^{150} \mathrm{Sn}$, calculated for the force SLy $4^{\rho+\delta \rho}$ and two different boundary conditions: Dirichlet (vanishing wave functions at $r=R_{\text {box }}$ ) or Neumann (vanishing derivative of the wave functions at $r=R_{\mathrm{box}}$ ).

\section{Examples}

\subsection{Self-consistent calculation for ${ }^{150} \mathrm{Sn}$}

As a first example we discuss here the results obtained for the semi magic isotope ${ }^{150} \mathrm{Sn}(N=100$ and $Z=50)$ using the effective force SLy $4^{\rho+\delta \rho}$. This nucleus is an interesting example since it has a rather small neutron Fermi energy $\left(\lambda_{N} \simeq-1.066 \mathrm{MeV}\right)$. Small neutron separation energy allows us to discuss possible effects of the integration box size as well as the choice of the boundary condition. If not explicitly stated otherwise, in all calculations the integration step is $h=0.2 \mathrm{fm}$ for the box size of $R_{\mathrm{box}}=30 \mathrm{fm}$, i.e., the number of integration points is $150+1$. The global properties of the nucleus for Dirichlet or Neumann boundary condition are shown in table 5.

In addition to the small neutron separation energy, one finds several single particle states with a significant occupation around $\lambda_{N}$ with different values of $\ell$ and indeed different asymptotic bahaviour. The single particle neutron states in the vicinity of the Fermi energy are shown in table 6 . It is gratifying to observe that despite the fact that the quasiparticle states are a little bit influenced by the change of the boundary conditions, the canonical states remain unchanged.

When a rather exotic nucleus is studied, it is important to check how well the results are converged with respect to the various approximations which are made in the numerical treatment of the HFB problem. First of all, we limit the calculation to a maximum number of partial wave given by $J \leq J_{\max }$, which has to by consistent with the mass of the nucleus. The convergence of the kinetic, pairing, and total energies are shown in the left part of Fig. 2. Between $2 J_{\max }=31$ and 37 the total energy difference is $1.3 \mathrm{keV}$. The changes in the kinetic and pairing energies are a little bit bigger $(7 \mathrm{keV}$ and $6 \mathrm{keV}$, respectively); since these quantities are not at their extrema, they react more rapidly to a small change of the wave function.

As discussed in section 5.3, the introduction of the cut-off radius $R_{\text {cut }}$ in the 


\begin{tabular}{|l|l|llll|}
\hline Boundary condition & $n \ell j$ & $3 p_{1 / 2}$ & $3 p_{3 / 2}$ & $2 f_{5 / 2}$ & $1 h_{9 / 2}$ \\
\hline Dirichlet & $E(\mathrm{MeV})$ & 0.926 & 0.937 & 1.330 & 1.556 \\
& $N$ & 0.221 & 0.577 & 0.254 & 0.438 \\
& $\varepsilon(\mathrm{MeV})$ & 1.169 & 1.051 & 1.445 & 1.572 \\
& $v^{2}$ & 0.251 & 0.619 & 0.264 & 0.438 \\
\hline Neumann & $E(\mathrm{MeV})$ & 0.919 & 0.932 & 1.328 & 1.556 \\
& $N$ & 0.211 & 0.562 & 0.251 & 0.438 \\
& $\varepsilon(\mathrm{MeV})$ & 1.168 & 1.051 & 1.445 & 1.572 \\
& $v^{2}$ & 0.251 & 0.619 & 0.263 & 0.438 \\
\hline
\end{tabular}

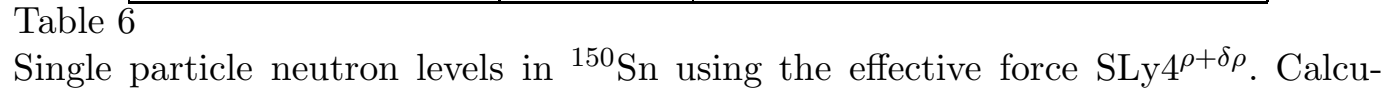
lations have been made in a $30 \mathrm{fm}$ box with an integration step of $0.2 \mathrm{fm}$. $E$ and $E$ are the quasiparticle energies and norms of the lower components while $\varepsilon$ is the canonical energy (54) and $v^{2}$ is the occupation factor of the corresponding canonical state (55).
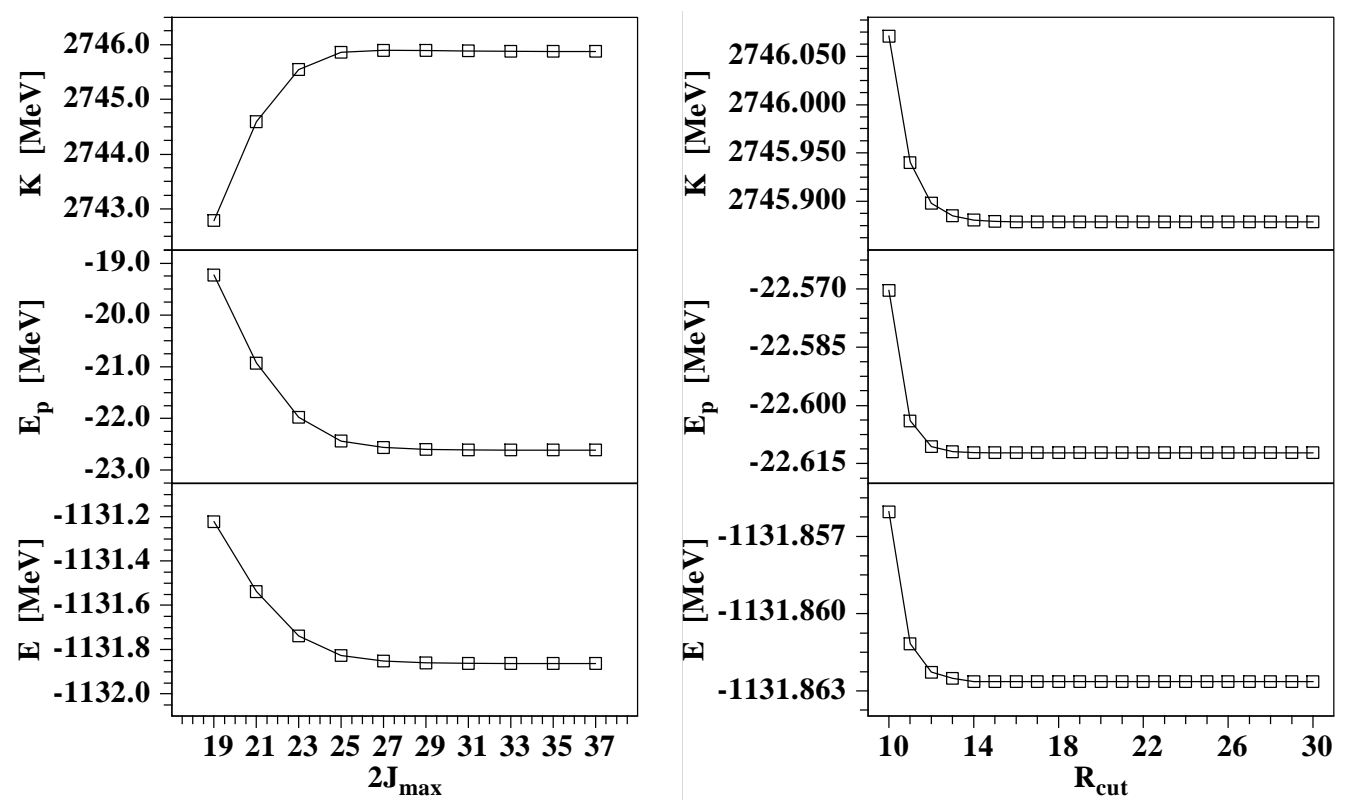

Fig. 2. Kinetic (top panels), pairing (middle panels) and total energies (lower panels) for ${ }^{150} \mathrm{Sn}$ as functions of $2 J_{\max }$ in a $30 \mathrm{fm}$ box with an integration step of $0.2 \mathrm{fm}$ (left) and of the pairing-field cut-off radius $R_{\text {cut }}$ defined in Eq. (77) (right).

pairing channel, Eq. (77), can improve the numerical stability of the iterative procedure when solving the HFB equation. The right part of Figure 2 displays the evolution of the kinetic, pairing, and total energies with the same parameters as in the left part of the Figure, except for the value of $2 J_{\max }$ which is fixed at 31 . It can be clearly seen that beyond a rather small distance, the 
total energy and its components are not affected by the introduction of this cut-off. Specifically, for $R_{\text {cut }} \geq 17 \mathrm{fm}$ the change in the total energy is less than $0.001 \mathrm{keV}$.

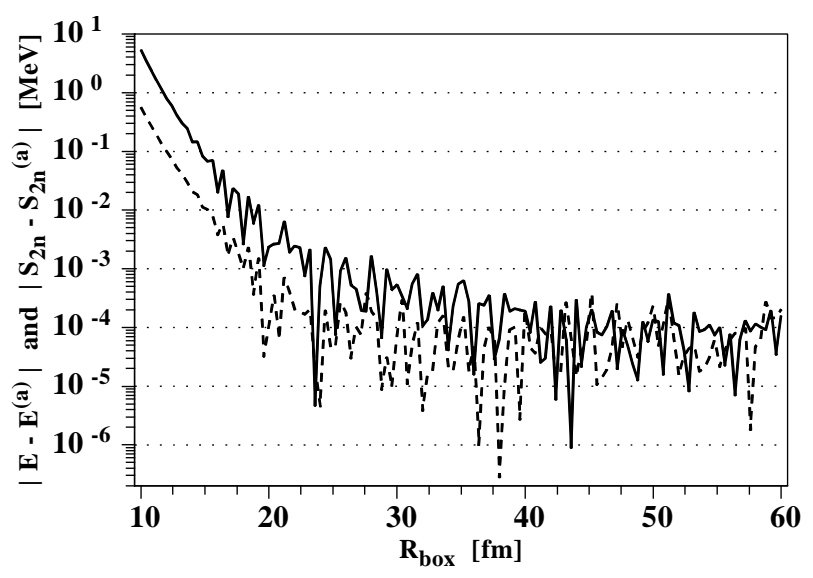

Fig. 3. Difference between the total energy $E$ of ${ }^{150} \mathrm{Sn}$ (solid line) and its average asymptotic value $E^{(a)}$ (see text) as a function of the box size $R_{\text {box }}$, and the analogous difference for the two-neutron separation energy $S_{2 n}$ (dashed line).

Figure 3 shows the convergence of the total energy $E$ and two-neutron separation energy $S_{2 n}$ as functions of the size of the box. Calculations have been made for $R_{\text {box }}$ ranging from $10 \mathrm{fm}$ to $60 \mathrm{fm}$ with a step of $0.4 \mathrm{fm}$. The cut-off equivalent energy of $E_{\text {cut }}=60 \mathrm{MeV}$ and the diffuseness of $1 \mathrm{MeV}$ have been used. In order to clearly exhibit the asymptotic trend of these quantities we have estimated their average asymptotic values $E^{(a)}$ and $S_{2 n}^{(a)}$. Since for $R_{\text {box }} \geq 35 \mathrm{fm}$ the results do not show any significant evolution, these values are estimated by taking averages for $35 \mathrm{fm} \leq R_{\text {box }} \leq 60 \mathrm{fm}$, which gives $E^{(a)}=-1131.862114 \mathrm{MeV}$ and $S_{2 n}^{(a)}=-1.925548 \mathrm{MeV}$. Here, the last significant digit corresponds to $0.001 \mathrm{keV}$, which is the accuracy required to stop the iterations when we solve the HFB equations for any given value of $R_{\mathrm{box}}$. The fluctuations around the average values result from the individual continuum states entering into the cut-off window with increasing $R_{\text {box }}$. We see that for relatively small boxes $(R \leq 20 \mathrm{fm})$ the two-neutron separation energy is an order of magnitude more stable than the total binding energy of the nucleus. For $R \geq 30 \mathrm{fm}$, the two quantities reach their asymptotic values with a random dispersion of about $0.5 \mathrm{keV}$. The value of $R_{\text {box }}=60 \mathrm{fm}$ is obviously unnecessarily large but it represents a good test of the stability of the integration procedure. From these results we conclude that the solution of the HFB equations with the box boundary conditions and the energy cut-off is precise up to about $1 \mathrm{keV}$.

A typical asymptotic behaviour of the particle and pairing densities for neutrons is shown in Fig. 4 for two different choices of the boundary condition. The impact of the boundary conditions only shows up within about $5 \mathrm{fm}$ near the box edge. It is small enough to have no significant effect on the calculated 
observables.

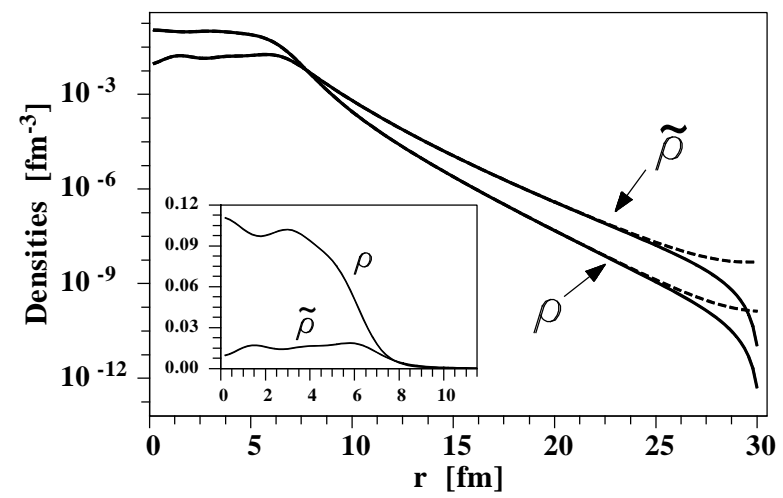

Fig. 4. Neutron particle and pairing densities in ${ }^{150} \mathrm{Sn}$ for Dirichlet (solid line) and Neumann (dashed line) boundary conditions. The inset represents the same quantities on a linear scale, in that case the impact of the boundary condition cannot be seen.

Neutron particle and pairing densities for different sizes of the box are shown in Fig. 5. The box radius of $10 \mathrm{fm}$ is obviously too small and it has only been included to show the effect of a very small box on the densities. For $R_{\text {box }} \geq 20 \mathrm{fm}$, no differences can be seen in the linear scale. An interesting fact is that despite the cut that has been applied for the pairing field at $R_{\text {cut }}=30 \mathrm{fm}$, see Sec. 5.3, no consequences can be observed on the densities in the asymptotic region.
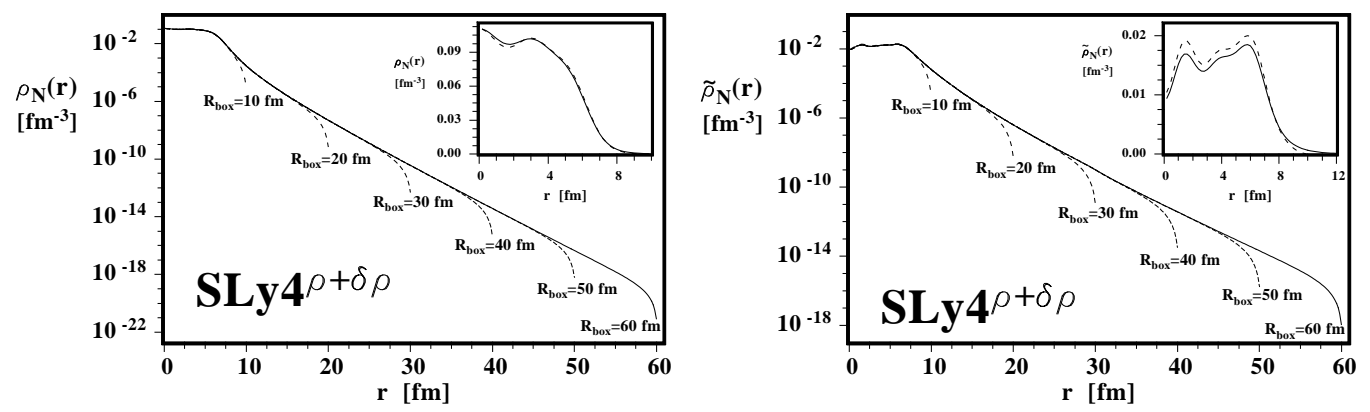

Fig. 5. Neutron particle (left) and pairing (right) densities for different box sizes. In the insets the dashed lines correspond to $R_{\text {box }}=10 \mathrm{fm}$ while the solid lines correspond to the other values of $R_{\text {box }}$ that cannot be separated in the linear scale.

\subsection{Regularization of the pairing field}

In this section, we present results obtained by using the regularization method proposed by A. Bulgac and Y. Yu [9]. Calculations have been made with the same box size and integration step as in the previous section, and the partial 
waves have been included up to $J=43 / 2$. The effective force used is SLy4, see table 1, combined with the mixed pairing force with parameters given in table 2. When evaluating the densities, contributions of quasiparticle states are included up to the maximum equivalent energy $E_{\max }$, see Eq. (47), but once this maximum energy is high enough the global properties of the nucleus do not depend on it.
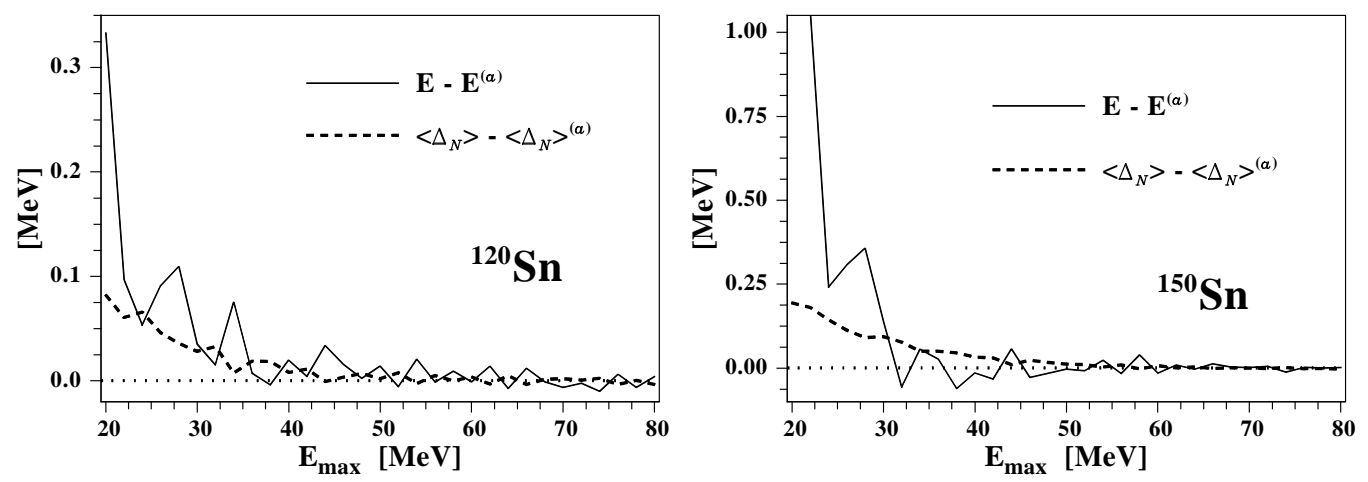

Fig. 6. Modulus of the difference between the total energy $E$ and its mean value $E^{(a)}$ (solid lines) and between the average neutron gap and its mean value (dashed lines) as functions of the energy $E_{\max }$ (see text) for ${ }^{120} \mathrm{Sn}$ (left) and ${ }^{150} \mathrm{Sn}$ (right). The mean values are calculated over the interval $60<E_{\max }<80 \mathrm{MeV}$.

It appears that the stability of results is very satisfying, even for a very exotic nucleus. This is shown in Figure 6, where the total energy and mean neutron gap are displayed as functions of $E_{\max }$ for ${ }^{120} \mathrm{Sn}$ and ${ }^{150} \mathrm{Sn}$. For $E_{\max }$ greater than $60 \mathrm{MeV}$, where the total energy does not show any significant evolution, we have evaluated its asymptotic limit $E^{(a)}$, and the analogous limit of the neutron mean pairing gap $\langle\Delta\rangle^{(a)}$, by averaging their respective values over the interval ranging from 60 to $80 \mathrm{MeV}$. The results are $E^{(a)}=1018.529 \mathrm{MeV}$ and $\langle\Delta\rangle^{(a)}=1.245 \mathrm{MeV}$ for ${ }^{120} \mathrm{Sn}$, and $E^{(a)}=1131.492 \mathrm{MeV}$ and $\langle\Delta\rangle^{(a)}=$ $1.499 \mathrm{MeV}$ for ${ }^{150} \mathrm{Sn}$. In this interval, the energies are scattered within $\pm 16 \mathrm{keV}$ and the gaps within $\pm 7 \mathrm{keV}$. Since the increase of $E_{\max }$ from $60 \mathrm{MeV}$ to $80 \mathrm{MeV}$ does not change the results in a significant way, the choice of $E_{\max }=$ $60 \mathrm{MeV}$ has been made for the rest of this study. In principle, this value should be readjusted in other mass region or when using a different effective force.

Within the cut-off prescription and regularization scheme we have calculated the series of even-even tin isotopes by using the SLy $4^{(\rho+\delta \rho)}$ force. The left part of Fig. 7 displays the binding energies per particle and the right part the deviation between the calculated binding energies and the experimental ones [25]. One can see that both methods give very similar results. The neutron mean gap are plotted on the left part of Fig. 8. In the two sets of calculation, the strengths of the pairing force have been adjusted in order to give the same gap in ${ }^{120} \mathrm{Sn}$. Again, we do not observe here any significant change when using or not the regularization scheme, although the gap is slightly reduced in heavy tin isotopes. Finally, the right part of Fig. 8 compares the differences between 

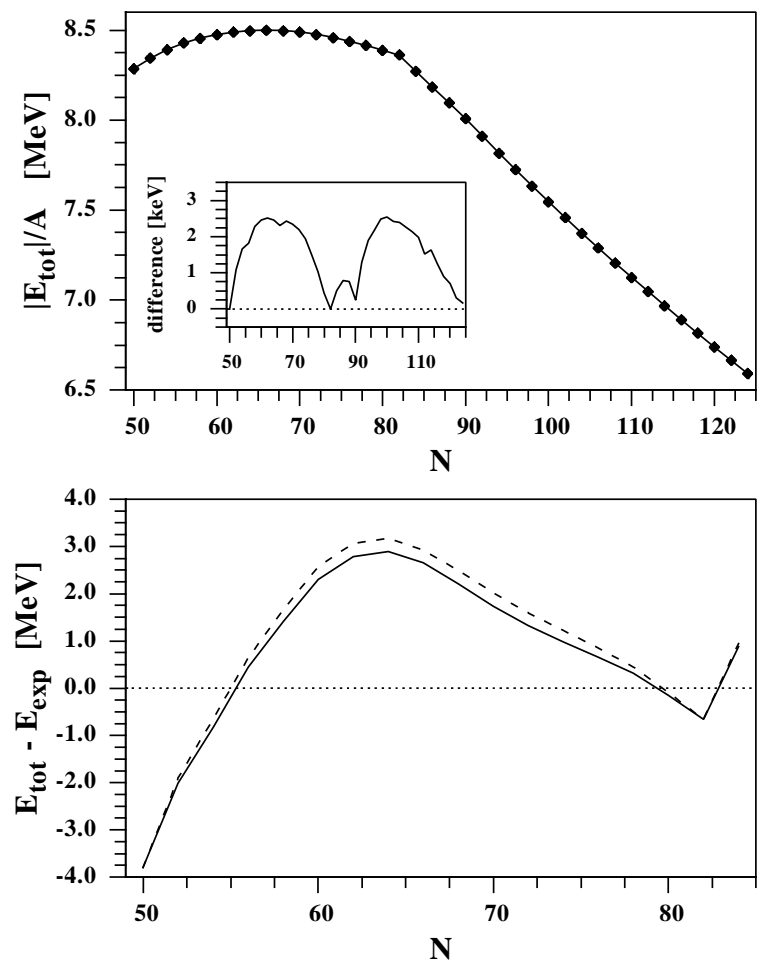

Fig. 7. Left figure: Binding energy per nucleon in tin isotopes. The solid line correspond to calculations with a cut-off energy of $E_{\text {cut }}=60 \mathrm{MeV}$ in the equivalent spectrum and the diamond symbols to the regularization of the energy with the summation of densities up to $E_{\max }=60 \mathrm{MeV}$ (see text). The difference between the two sets of results is plotted in the inset. Right figure: Differences between the calculated and measured binding energies shown for those tin isotopes for which the data are known; solid line corresponds to the introduction of a cut-off and the dashed line to the regularization.

the neutron and proton rms radii plotted with respect to that in ${ }^{132} \mathrm{Sn}$; once again the two methods give extremely similar results.

\subsection{Neutron drip line}

As a last example we consider the two-neutron drip line for $Z=48$. The case of $Z=50$ is not interesting for our example because there the neutron pairing correlations vanish at the two-neutron drip line (at least for the pairing force considered here), so the residual coupling between the bound and scattering states disappears. The two-neutron drip line is defined by the vanishing Fermi energy, $\lambda_{N}=0$. In such a system, the HFB approximation leads to a fully continuous quasiparticle spectrum and the minimum quasiparticle energy is determined by the pairing correlations only [26]. In this extreme situation it is important to check if the box boundary conditions have any significant effect on the results. To this end, we have performed calculations with the box 

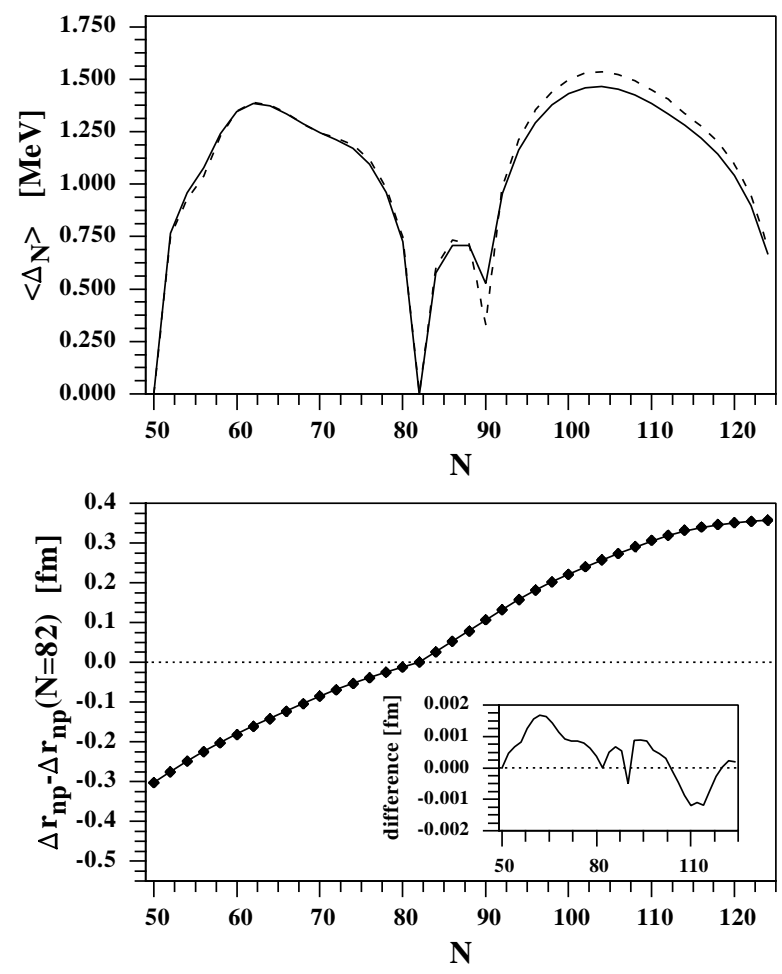

Fig. 8. Left figure: Mean neutron gap in tin isotopes, the solid line corresponds to the introduction of a cut-off and the dashed line to the regularization. Right figure: Differences between the rms radii of neutrons and protons in tin isotopes compared with the same quantity for ${ }^{132} \mathrm{Sn}$, the solid line corresponds to the introduction of a cut-off and the diamond symbol to the regularization, while the difference between the two sets is plotted in the inset.

size $R_{\text {box }}$ varying between 10 and $35 \mathrm{fm}$ and with the regularized pairing field corresponding to $E_{\max }=60 \mathrm{MeV}$.
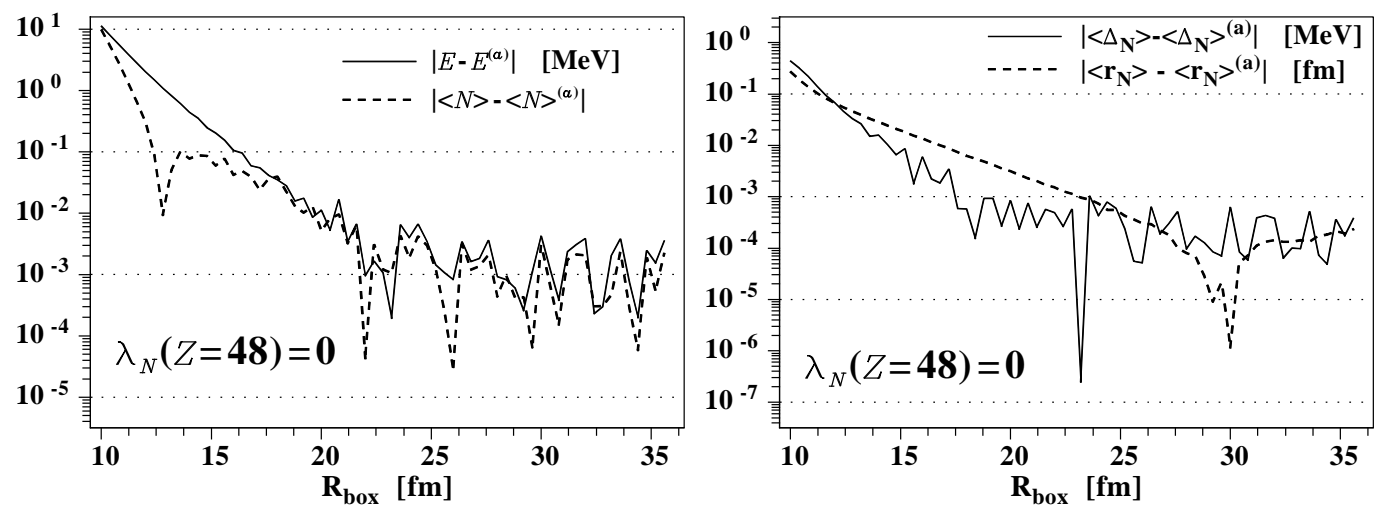

Fig. 9. Differences between the total energy, number of neutrons, neutron rms radius, and neutron pairing gap, and their corresponding average asymptotic values (see text), calculated for the $Z=48$ two-neutron drip line nucleus.

We used the same method as in Sec. 8.1 to estimate the average asymptotic values (for $R_{\text {box }} \geq 25 \mathrm{fm}$ ) of the total energy $E^{(a)}=-1095.400501 \mathrm{MeV}$, neutron 
number $\langle n\rangle^{(a)}=117.053163$, pairing gap $\left\langle\Delta_{N}\right\rangle^{(a)} 1.150396 \mathrm{MeV}$, and neutron rms radius $\left\langle r_{N}\right\rangle^{(a)}=5.557959 \mathrm{fm}$. Differences of these observables relative to the above average values are reported in Fig. 9. It is seen that the energy is stable up to several $\mathrm{keV}$ once the radius of the box is bigger than $25 \mathrm{fm}$, and no further significant change is obtained by enlarging it. The mean neutron number is very stable too, despite the large spatial extension of the neutron density in such a drip line nucleus. As discussed in Ref. [26], the appearance of a giant neutron halo is prevented by the pairing correlations (the pairing anti-halo effect) and the neutron rms radius is perfectly stable with increasing box size. The same is also true for the neutron pairing gap.

\section{Conclusion}

The code HFBRAD (v1.00) which we introduced in this paper allows for a very rapid determination of self-consistent spherical ground states of nuclei. It treats the pairing correlations within the HFB method and implements the regularization of the divergent part of the pairing energy, and hence is a perfect tool to study nuclei near drip lines.

Realistic calculations must, of course, take into account the effects of the deformation, and the codes that are able to perform such tasks have also been published in Refs. $[1,2]$. However, deformed calculations are generally much more time consuming and solutions within spherical symmetry will always remain very useful, not only for test purposes, but also whenever results for many different values of input parameters are called for. This later aspect becomes essential when fitting of the force parameters has to be performed, which will constitute the main future application of the present code.

\section{Acknowledgments}

We would like to thank A. Bulgac and N. Sandulescu for useful discussions during the development of the code HFBRAD. This work was supported in part by the Polish Committee for Scientific Research (KBN) under Contract No. 1 P03B 05927 and by the Foundation for Polish Science (FNP); and by the U.S. Department of Energy under Contract Nos. DE-FG02-96ER40963 (University of Tennessee), DE-AC05-00OR22725 with UT-Battelle, LLC (Oak Ridge National Laboratory), and DE-FG05-87ER40361 (Joint Institute for Heavy Ion Research). 


\section{References}

[1] J. Dobaczewski and J. Dudek, Comput. Phys. Commun. 102, 166 (1997); 102, 183 (1997), 131, 164 (2000), J. Dobaczewski and P. Olbratowski, ibid. 158, 158 (2004).

[2] M.V. Stoitsov, J. Dobaczewski, W. Nazarewicz, and P. Ring, to be published in Computer Physics Communication.

[3] D. Vautherin and D.M. Brink, Phys. Rev. C5, 626 (1972).

[4] M. Beiner, H. Flocard, N. Van Giai, and P. Quentin, Nucl. Phys. A238, 29 (1975).

[5] J. Dobaczewski, H. Flocard and J. Treiner, Nucl. Phys. A422, 103 (1984).

[6] P.-G. Reinhard, in Computational Nuclear Physics 1 K. Langanke, J.A. Maruhn, and S.E. Koonin, eds. (Springer Verlag, Berlin, 1991) p.28.

[7] W. Pöschl, D. Vretenar, and P. Ring, Comput. Phys. Commun. 103, 217 (1997).

[8] M. Grasso, N. Van Giai, and N. Sandulescu, Phys. Lett. B 535, 103 (2002).

[9] A. Bulgac and Y. Yu, Phys. Rev. Lett. 88, 042504 (2002).

[10] P. Ring and P. Schuck, The Nuclear Many-Body Problem (Springer-Verlag, Berlin, 1980).

[11] Y.M. Engel, D.M. Brink, K. Goeke, S.J. Krieger, and D. Vautherin, Nucl. Phys. A249, 215 (1975).

[12] K. Bennaceur, J. Dobaczewski, and M. Płoszajczak, Phys. Lett. B496, 154 (2000).

[13] J. Dobaczewski and J. Dudek, Phys. Rev. C52, 1827 (1995).

[14] J.W. Negele and D. Vautherin, Phys. Rev. C5, 1472 (1972).

[15] C. Titin-Schnaider and P. Quentin, Phys. Lett. 49B, 397 (1974).

[16] J. Skalski, Phys. Rev. C63, 024312 (2001).

[17] A. Bulgac, Preprint FT-194-1980, Central Institute of Physics, Bucharest, 1980; nucl-th/9907088.

[18] J. Dobaczewski, W. Nazarewicz, T.R. Werner, J.-F. Berger, C.R. Chinn, and J. Dechargé, Phys. Rev. C53, 2809 (1996).

[19] A. Bulgac and Y. Yu, Beijing International Summer School on Subatomic Physics, 2001 and International Workshop on Nuclear Physics with RIB, Lanzhou, 2001, nucl-th/0109083.

[20] M. Bender, P.-H. Heenen, and P.-G. Reinhard, Rev. Mod. Phys. 75, 121 (2003). 
[21] J. Bartel, P. Quentin, M. Brack, C. Guet, and H.B. Håkansson, Nucl. Phys. A386, 79 (1982).

[22] E. Chabanat, P. Bonche, P. Haensel, J. Meyer, and F. Schaeffer, Nucl. Phys. A635, 231 (1998).

[23] G. Dahlquist and A. Björck, Numerical Methods, (Prentice-Hall, Engelwoods Cliffs, 1974).

[24] S.T. Belyaev, A.V. Smirnov, S.V. Tolokonnikov, and S.A. Fayans, Sov. J. Nucl. Phys. 45, 783 (1987).

[25] G. Audi, A.H. Wapstra, and C. Thibault, Nucl. Phys. A729, 337 (2003).

[26] K. Bennaceur, J. Dobaczewski, and M. Płoszajczak, Phys. Rev. C60, 034308 (1999). 


\section{TEST RUN INPUT}

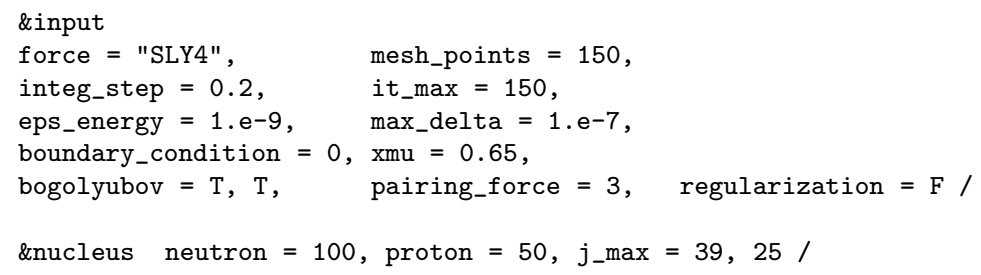




\section{TEST RUN OUTPUT}

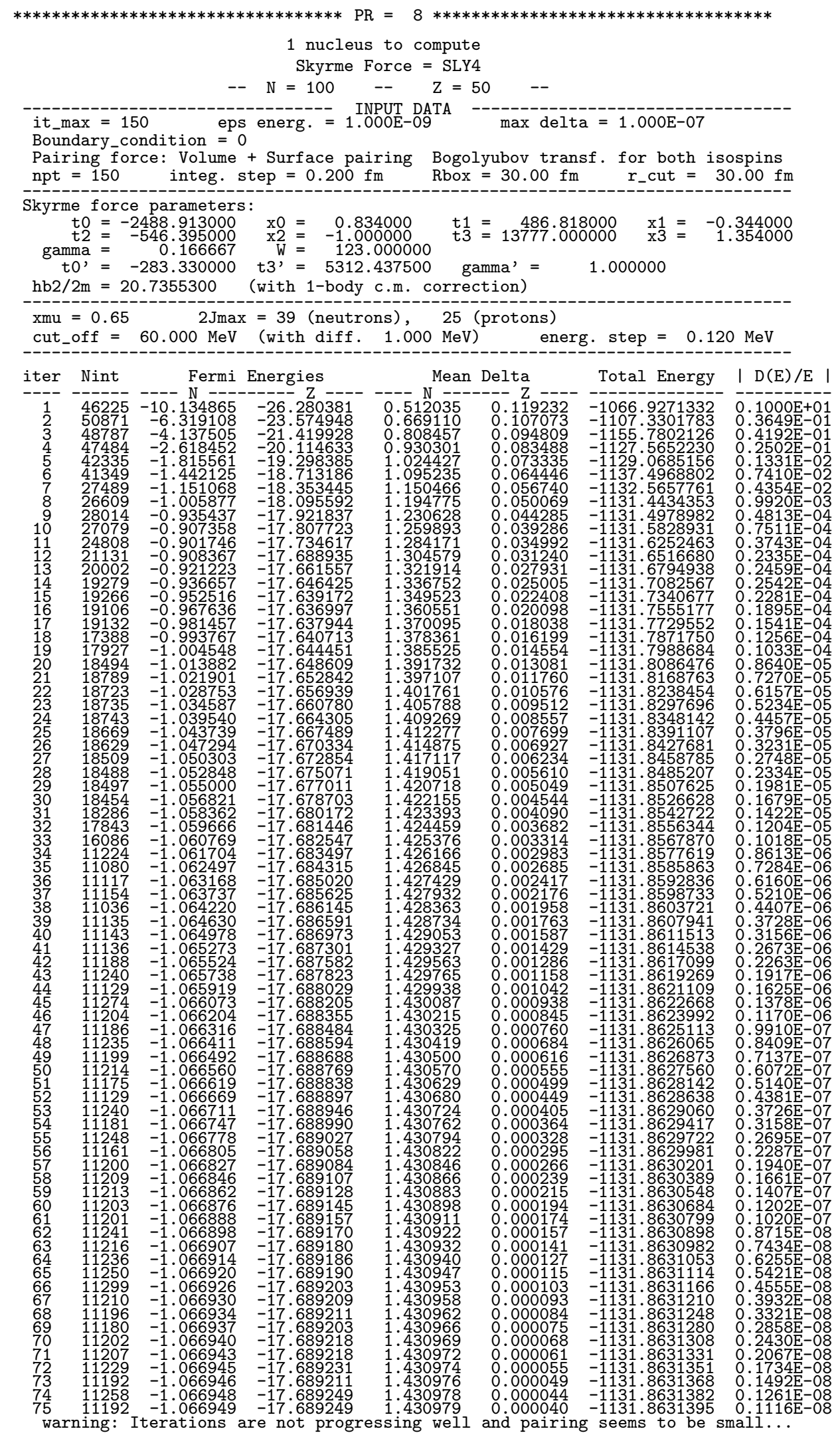


Trying to force convergence for it $=2$

\begin{tabular}{|c|c|c|c|c|c|c|c|}
\hline $\begin{array}{l}76 \\
77 \\
78 \\
79 \\
80 \\
81 \\
82 \\
83 \\
84 \\
85 \\
86 \\
87 \\
88 \\
89\end{array}$ & $\begin{array}{l}11211 \\
14395 \\
14316 \\
14417 \\
11225 \\
14323 \\
14242 \\
11168 \\
11225 \\
14356 \\
14393 \\
11269 \\
14414 \\
14353\end{array}$ & $\begin{array}{l}\text {-1.066950 } \\
-1.066951 \\
-1.066952 \\
-1.066953 \\
-1.066953 \\
-1.066954 \\
-1.066954 \\
-1.066955 \\
-1.066955 \\
-1.066955 \\
-1.066955 \\
-1.066956 \\
-1.066956 \\
-1.066956\end{array}$ & $\begin{array}{l}-17.652243 \\
-17.648398 \\
-17.653151 \\
-17.653151 \\
-17.631479 \\
-17.640449 \\
-17.640449 \\
-17.640449 \\
-17.623581 \\
-17.581957 \\
-17.581957 \\
-17.645585 \\
-17.606532 \\
-17.606532\end{array}$ & $\begin{array}{r}1.430981 \\
1.430982 \\
1.430983 \\
1.430983 \\
1.430984 \\
1.430985 \\
1.430985 \\
1.430986 \\
1.430986 \\
1.430986 \\
1.430987 \\
1.430987 \\
1.430987 \\
1.430987\end{array}$ & $\begin{array}{l}0.000002 \\
0.000001 \\
0.000001 \\
0.000001 \\
0.000001 \\
0.000001 \\
0.000001 \\
0.000001 \\
0.000001 \\
0.000001 \\
0.000001 \\
0.000001 \\
0.000000 \\
0.000000\end{array}$ & $\begin{array}{l}-1131.8631406 \\
-1131.8631415 \\
-1131.8631421 \\
-1131.8631428 \\
-1131.8631434 \\
-1131.8631438 \\
-1131.8631442 \\
-1131.8631445 \\
-1131.8631449 \\
-1131.8631452 \\
-1131.8631454 \\
-1131.8631455 \\
-1131.8631457 \\
-1131.8631459\end{array}$ & $\begin{array}{l}0.9561 \mathrm{E}-09 \\
0.7948 \mathrm{E}-09 \\
0.5991 \mathrm{E}-09 \\
0.6288 \mathrm{E}-09 \\
0.4951 \mathrm{E}-09 \\
0.3779 \mathrm{E}-09 \\
0.3551 \mathrm{E}-09 \\
0.2583 \mathrm{E}-09 \\
0.3381 \mathrm{E}-09 \\
0.2942 \mathrm{E}-09 \\
0.1300 \mathrm{E}-09 \\
0.1205 \mathrm{E}-09 \\
0.1428 \mathrm{E}-09 \\
0.1478 \mathrm{E}-09\end{array}$ \\
\hline iter & Nint & Fermi & Energies & Mean & Delta & Total Energy & $|D(E) / E|$ \\
\hline 90 & 11162 & -1.066956 & -17.486178 & $-\overline{1.430987}$ & 0.000000 & -1131.8631460 & $0.1546 \mathrm{E}-09$ \\
\hline $\begin{array}{l}\text { Fermi } \\
\text { Mean } \\
\text { Kinet } \\
\text { Pairi } \\
\text { Pair. }\end{array}$ & $\begin{array}{l}\text { Ener. } \\
\text { Gaps } \\
\text { ic En. } \\
\text { ng En. } \\
\text { Kin.En. }\end{array}$ & $\begin{array}{r}-1 . \\
-1 . \\
1987 . \\
-22 . \\
0 .\end{array}$ & $\begin{array}{l}6695605 \\
3098737 \\
30592234 \\
1435165 \\
0000000\end{array}$ & $\begin{array}{r}\text { Protons } \\
17.48617778 \\
-0.00000038 \\
58.56803134 \\
0.00000000 \\
0.00000000\end{array}$ & $\begin{array}{r}2745.873 \\
-22.614 \\
0.000\end{array}$ & $\begin{array}{l}95368 \\
35165 \\
00000\end{array}$ & \\
\hline $\begin{array}{l}\text { E/A } \\
\text { Contr } \\
\text { Fiel } \\
\text { (Rear }\end{array}$ & $\begin{array}{l}\text { ies (in } \\
=\quad-7.5 \\
\text { ibution }\end{array}$ & $\begin{array}{l}18.250650 \\
77.050463)\end{array}$ & $\begin{array}{l}\text { ariat. }= \\
\text { Spin-Or. = }\end{array}$ & -67.645555 & $\begin{array}{c}====>\text { Et } \\
\text { Coul. = } \\
\text { Coul.Ex. = }\end{array}$ & $\begin{array}{l}\text { ot }=-1131.8631 \\
349.004642 \\
-18.231185\end{array}$ & \\
\hline & & $\begin{array}{r}\text { Neu } \\
100.0 \\
5.2\end{array}$ & $\begin{array}{l}\text { ons } \\
000 \\
562\end{array}$ & $\begin{array}{l}\text { rotons } \\
.000000 \\
.820502\end{array}$ & Total & Charge & \\
\hline
\end{tabular}

